\title{
Identifying an early treatment window for predicting breast cancer response to neoadjuvant chemotherapy using immunohistopathology and hemoglobin parameters
}

\author{
Quing Zhu ${ }^{1 *}$, Susan Tannenbaum² ${ }^{2}$ Scott H. Kurtzman ${ }^{3}$, Patricia DeFusco ${ }^{4}$, Andrew Ricci Jr ${ }^{4}$, Hamed Vavadi ${ }^{5}$,
} Feifei Zhou ${ }^{5}$, Chen Xu' ${ }^{6}$, Alex Merkulov², Poornima Hegde ${ }^{2}$, Mark Kane ${ }^{2}$, Liqun Wang ${ }^{7}$ and Kert Sabbath ${ }^{3}$

\begin{abstract}
Background: Breast cancer pathologic complete response ( $\mathrm{PCR}$ ) to neoadjuvant chemotherapy $(N A C)$ varies with tumor subtype. The purpose of this study was to identify an early treatment window for predicting pCR based on tumor subtype, pretreatment total hemoglobin ( $\mathrm{tHb}$ ) level, and early changes in $\mathrm{tHb}$ following NAC.

Methods: Twenty-two patients (mean age 56 years, range 34-74 years) were assessed using a near-infrared imager coupled with an Ultrasound system prior to treatment, 7 days after the first treatment, at the end of each of the first three cycles, and before their definitive surgery. Pathologic responses were dichotomized by the Miller-Payne system. Tumor vascularity was assessed from $\mathrm{tHb}$; vascularity changes during NAC were assessed from a percentage tHb normalized to the pretreatment level (\%tHb). After training the logistic prediction models using the previous study data, we assessed the early treatment window for predicting pathological response according to their tumor subtype (human epidermal growth factor receptor 2 (HER2), estrogen receptor (ER), triple-negative (TN)) based on $\mathrm{HH}$, and \%tHb measured at different cycles and evaluated by the area under the receiver operating characteristic (ROC) curve (AUC).

Results: In the new study cohort, maximum pretreatment $\mathrm{tHb}$ and \%tHb changes after cycles 1, 2, and 3 were significantly higher in responder Miller-Payne $4-5$ tumors $(n=13)$ than non-or partial responder Miller-Payne 1-3 tumors $(n=9)$. However, no significance was found at day 7 . The AUC of the predictive power of pretreatment $\mathrm{HH}$ in the cohort was 0.75 , which was similar to the performance of the HER2 subtype as a single predictor (AUC of 0.78 ). A greater predictive power of pretreatment tHb was found within each subtype, with AUCS of 0.88, 0.69, and 0.72, in the HER2, ER, and TN subtypes, respectively. Using pretreatment tHb and cycle $1 \% \mathrm{HHb}$, AUC reached 0.96, 0.91, and $0.90 \mathrm{in} \mathrm{HER} 2$, ER, and TN subtypes, respectively, and 0.95 regardless of subtype. Additional cycle $2 \% \mathrm{tHb}$ measurements moderately improved prediction for the HER2 subtype but did not improve prediction for the ER and TN subtypes.
\end{abstract}

Conclusions: By combining tumor subtypes with $\mathrm{HH}$, we predicted the $\mathrm{pCR}$ of breast cancer to NAC before treatment. Prediction accuracy can be significantly improved by incorporating cycle 1 and $2 \%$ tHb for the HER2 subtype and cycle 1 $\% \mathrm{HHb}$ for the ER and TN subtypes.

(Continued on next page)

\footnotetext{
* Correspondence: zhu.q@wustl.edu

${ }^{1}$ Biomedical Engineering and Radiology, Washington University in St Louis, One Brookings Drive, Mail Box 1097, Whitaker Hall 300D, St. Louis, MO 63130, USA

Full list of author information is available at the end of the article
}

(c) The Author(s). 2018 Open Access This article is distributed under the terms of the Creative Commons Attribution 4.0 International License (http://creativecommons.org/licenses/by/4.0/), which permits unrestricted use, distribution, and reproduction in any medium, provided you give appropriate credit to the original author(s) and the source, provide a link to the Creative Commons license, and indicate if changes were made. The Creative Commons Public Domain Dedication waiver (http://creativecommons.org/publicdomain/zero/1.0/) applies to the data made available in this article, unless otherwise stated. 
(Continued from previous page)

Trial registration: ClinicalTrials.gov, NCT02092636. Registered in March 2014.

Keywords: Predicting neoadjuvant chemotherapy, Personalized medicine, Near infrared imaging, Ultrasound-guided optical imaging,

\section{Background}

The increasingly widespread use of neoadjuvant chemotherapy (NAC) in breast cancer patients has improved surgical outcomes by preoperatively downsizing the tumor volume. Since the introduction of NAC, breastconserving surgery rates have increased [1]. Moreover, patients who have achieved pathological complete response (pCR) show improved survival rates compared with those who did not achieve pCR [2]. This relationship is so strong, in fact, that pCR is becoming a surrogate endpoint for evaluating the effectiveness of newer chemotherapy protocols [3, 4]. Early assessment of the degree of patient response to NAC can have a major impact on individualized treatment management [5].

The ability to identify patients with tumors that have a high likelihood of achieving a pCR before starting NAC could enable targeting a treatment plan to patients with only those tumor types. By eliminating ineffective treatment of patients unlikely to benefit, outcomes would be better, and toxicity would be reduced. Published literature shows that an increased probability of achieving pCR is correlated with high tumor grade, positive human epidermal growth factor receptor 2 (HER2) status, negative estrogen receptor (ER) status, and triple negative (TN) receptor status [6-9]. However, prediction models based on these tumor histopathological characteristics are imperfect; within and among these subgroups, the response to chemotherapy varies widely. Dual HER2 blockade with trastuzumab and pertuzumab in combination with cytotoxic chemotherapy now utilized in many $\mathrm{HER}^{+}$patients in the neoadjuvant setting results in a high pCR (16.8-66.2\%) [10]. Despite this, there is a significant percentage of $\mathrm{HER} 2^{+}$patients who do not achieve a pCR or near pCR. Additionally, the $\mathrm{pCR}$ of $\mathrm{ER}^{+} / \mathrm{HER} 2^{-}$cancers is less robust $(7.0-16.2 \%)$ and the pCR for TN cancers is 33.6-35\% [11, 12].

Many ongoing investigations are exploring imaging techniques to monitor response and allow early modification of treatment in order to enhance outcomes [13]. The use of imaging as an early surrogate biomarker of response is appealing because it is noninvasive and might allow for a window of opportunity during which treatment regimens could be altered accordingly, depending on the expected response.

Conventional imaging methods, such as mammography and ultrasound (US), to monitor NAC have not been widely used to date due to their low sensitivity for monitoring NAC-treated tumors [14]. Positron emission tomography (PET)/computed tomography $(\mathrm{CT})$ is more sensitive to tumor metabolic activity which has been shown to be an early indicator of treatment effectiveness for breast cancer in the neoadjuvant setting [15, 16]. Contrast-enhanced magnetic resonance imaging (MRI) is effective in predicting $\mathrm{TN}$ or $\mathrm{HER}^{+}$cancers, but is inaccurate for $\mathrm{ER}^{+} / \mathrm{HER} 2^{-}$breast cancers [17]. Both $\mathrm{PET} / \mathrm{CT}$ and MRI require the injection of contrast agents and are costly for repeated use during treatment.

Optical tomography and spectroscopy using near infrared (NIR) diffused light have been explored as novel tools to predict and monitor the tumor vasculature response to NAC [18-31]. The NIR technique utilizes the intrinsic biomarker of hemoglobin contrast, which is directly related to tumor angiogenesis. Cost effectiveness, portability, and the absence of the need for contrast agents make NIR systems ideal for repeated use in clinical settings. We have reported the development of USguided optical tomography using NIR diffused light coupled with a commercial ultrasound system (NIR/US) to improve light localization and quantification accuracy in the diagnosis of breast cancer $[32,33]$ and in predicting NAC response [20]. The logistic prediction models we developed utilize tumor pretreatment pathological parameters and hemoglobin content measured before NAC to predict pathological response [21]. The present study was designed to identify the best treatment window for predicting pathological response during NAC using breast cancer subtype, the pretreatment biomarker of total hemoglobin (tHb) level, and changes in tHb during early-treatment cycles 1,2 , and 3 . Ultimately, effectively predicting the response to NAC by combining information from US-guided NIR with breast cancer subtype could help to individualize treatment.

\section{Methods \\ Patients}

The study protocol was approved by institutional review boards and was HIPAA compliant. Written informed consent was obtained from all patients. From March 2014 to June 2016, 28 patients were recruited at three hospitals. All had been referred for NAC to the one of three medical oncologists (PD, ST, and KS) and agreed to participate in our study. Five patients did not complete the study because of a change in their treatment plan or a desire to withdraw from the study. One 
patient had technically problematic baseline imaging. Data from these six patients were not included in the analysis. The remaining 22 patients (mean age 55 years, range 34-74 years) were repeatedly imaged by NIR/US prior to initiation of NAC, at the end of the first three treatment cycles during chemotherapy, and prior to definitive surgery. Of the 22 patients (Table 1), 11 were $\mathrm{HER}^{+}$of which eight were also $\mathrm{ER}^{+}$; six were $\mathrm{ER}^{+}$/ HER2 ${ }^{-}$, and five were TN. Of the 22 patients, 12 had T2 tumors, five had T1, and five had T3 tumors. Patients were treated with regimens based on their tumor biomarkers according to current clinical practice. For $\mathrm{ER}^{+} / \mathrm{HER} 2^{-}$tumors, patients were treated with dosedense doxorubicin/cyclophosphamide every 2 weeks for four cycles followed by paclitaxel every 2 weeks for four cycles (ACT). The NIR/US cycle 1 to 3 measurements were performed at the end of the first three cycles before the paclitaxel started. For $\mathrm{HER}^{+}$tumors, all patients were treated with trastuzumab, pertuzumab, and docetaxel or paclitaxel with or without carboplatin (TPT) every 3 weeks for six cycles and one patient had two additional cycles of 5-flurouracil, epirubicin, and cyclophosphamide (FEC). The NIR/US cycle 1 to 3 measurements were performed at the end of the first three treatment cycles when TPT was given. For TN tumors, three were treated with $\mathrm{ACT}$, the same as $\mathrm{ER}^{+} / \mathrm{HER}^{-}$ patients, and two were treated with carboplatin and paclitaxel every 3 weeks for six cycles because of their BRCA1 gene mutation. One elderly $\mathrm{ER}^{+} / \mathrm{HER} 2^{-}$patient was treated with cyclophosphamide/docetaxel without doxorubicin (TC) every 3 weeks for four cycles.

The HER2 ${ }^{+}$cohort was monitored at an additional time point of 7 days after the first treatment, and one $\mathrm{TN}$ and four $\mathrm{ER}^{+} / \mathrm{HER}^{-}$patients were also monitored

Table 1 Patient information, tumor characteristics, Miller-Payne Grade, initial tumor size (MRI/PET and US), and treatment regimen

\begin{tabular}{|c|c|c|c|c|c|c|c|c|c|}
\hline Age & Tumor type & NS & Mitotic count/10 HPF & TN & HER2/ER & Miller-Payne grade & Tumor size (MRI/PET) & Tumor size (US) & $\begin{array}{l}\text { Treatment } \\
\text { regimen }\end{array}$ \\
\hline 59 & IDC & 6 & 2 & - & $-/+$ & 3 & 2.7 & 1.7 & $\mathrm{ACT}$ \\
\hline 55 & ILC & 6 & 1 & - & $-/+$ & 4 & 8.6 & $-{ }^{a}$ & $\mathrm{ACT}$ \\
\hline 33 & IDC & 4 & 2 & - & $-/+$ & 3 & 1.6 & 1.5 & $\mathrm{ACT}$ \\
\hline 61 & IDC/ILC & 6 & 1 & + & $-/-$ & 1 & 3.3 & 1.9 & $\mathrm{ACT}$ \\
\hline 59 & IDC & 6 & 5 & - & $-/+$ & 2 & 5.6 & 2.2 & $\mathrm{ACT}$ \\
\hline 68 & IDC & 6 & 3 & - & $-/+$ & 3 & 7.6 & 2.2 & $\mathrm{ACT}$ \\
\hline 51 & IDC & 9 & 40 & + & $-1-$ & 5 & 3.6 & 2.2 & $\mathrm{ACT}$ \\
\hline \multirow[t]{2}{*}{53} & IDC & 9 & 62 & + & $-1-$ & 3 & PET: 3.7 & 4.3 & $\mathrm{ACT}$ \\
\hline & & & & & & & & & TPT \\
\hline 51 & IDC & 7 & 15 & - & $+/+$ & 5 & N/A & 1.2 & TPT \\
\hline 74 & IDC & 8 & 8 & - & $+/-$ & 5 & 6.2 & $--^{a}$ & TPT \\
\hline \multirow[t]{2}{*}{57} & IDC & 9 & 16 & - & $+/-$ & 4 & 6.9 & $-{ }^{b}$ & TPT\&FEC \\
\hline & & & & & & & & & TPT \\
\hline 51 & IDC & 9 & 14 & - & $+/-$ & 5 & 3.0 & 1.9 & TPT \\
\hline 59 & IDC & 7 & 1 & - & $+/+$ & 5 & N/A & 0.6 & TPT \\
\hline 61 & IDC & 4 & 3 & - & $+/+^{c}$ & 5 & 2.0 & 1.4 & TPT \\
\hline 37 & IDC/ILC & 8 & 9 & - & $+/+$ & 4 & 3.6 & 1.9 & TPT \\
\hline 54 & IDC & 8 & 20 & - & $+/+$ & 5 & 2.9 & 2.3 & TPT \\
\hline 40 & IDC & 8 & 15 & - & $+/+$ & 5 & 2.2 & 2.1 & TPT \\
\hline 62 & IDC & 9 & 12 & - & $+/+$ & 3 & PET: 1.8 & 1.2 & TPT \\
\hline 37 & IDC & 8 & 42 & - & $+/+^{c}$ & 5 & 1.5 & 1.6 & TPT \\
\hline 72 & IDC & 9 & 42 & - & $-/+$ & 3 & 4.3 & 2.3 & TC \\
\hline 57 & IDC & 9 & 14 & + & $-1-$ & 5 & 2.3 & 1.3 & Carbo/T \\
\hline 41 & IDC & 9 & 8 & + & $-/-$ & 3 & 4.0 & 4.0 & Carbo/T \\
\hline
\end{tabular}

${ }^{\mathrm{a}}$ Not US visible

${ }^{\mathrm{b}}$ Much larger than the size of the US transducer

'ER showed a weak positive result

ACT, dose-dense doxorubicin/cyclophosphamide and paclitaxel; Carbo/T, carboplatin and paclitaxel, ER estrogen receptor, FEC 5flurouracil, epirubicin, and cyclophosphamide, HER2 human epidermal growth factor receptor 2, IDC invasive ductal carcinoma, ILC invasive lobular carcinoma, MRI magnetic resonance imaging, N/A not available, NS Nottingham Score (out of 9), PET positron emission tomography, TC cyclophosphamide and docetaxel, TN triple negative, TPT TP and taxane-based therapy-trastuzumab, pertuzumab and docetaxel or paclitaxel with or without carboplatin, TPT\&FEC trastuzumab, pertuzumab, paclitaxel; 5flurouracil, epirubicin, cyclophosphamide, US ultrasound 
at an optional time point of 7 days after the first treatment. The median from 7 days was 0 with a range of 0 to 2 days. Moreover, four TN and five $\mathrm{ER}^{+} / \mathrm{HER} 2^{-}$ patients were monitored at an additional time point at the end of cycle 5. Thus, a total of 16 patients had an additional time point at 7 days after the first treatment and nine patients had an additional time point at the end of cycle 5.

All 22 patients were studied after their diagnostic core biopsy with an average interval of 28 days (median 26 days, range 7-56 days). Among the 22 patients, one had pretreatment NIR measurements 7 days after biopsy and the remaining patients had the NIR measurements more than 10 days after biopsy. All patients received the first cycle of NAC after the initial NIR/US study (median 2 days, range $0-10$ days). The average interval between post-treatment NIR/US and surgery was 19 days (median 15 days, range 2-67 days). During the treatment, the NIR/US scans were performed before their scheduled chemotherapy (median 0 days, range $0-5$ days). Among the 22 patients, 18 patients had pretreatment MRI and 12 patients had post-treatment MRI. Two patients had pretreatment PET.

The histologic type of 19 patients was invasive ductal carcinoma; one patient had invasive lobular carcinoma, and the other two patients had invasive mammary carcinoma with mixed ductal and lobular features. One of the 22 patients had two distinct tumor masses in the same breast, adjacent to each other and with the same histologic characteristics. For this patient, one of the two masses was used for data analysis. Invasive carcinoma within the pretreatment core biopsies was graded using the Nottingham histologic score (NS). ER, progesterone receptor (PR), and HER-2/neu (c-erbB-2) immunohistochemistry was performed on formalin-fixed, paraffin-embedded core biopsy tissue. The ER and PR were scored by a modified San Antonio scoring system [34], where the total score ranges from 0 to 8 (scores $0-2$ are negative, a score of 3 is equivocal and scores $\geq 4$ are positive). Testing for the HER2 gene was performed by immunohistochemistry and by gene amplification utilizing the fluorescence in situ hybridization (FISH) technique, and the results were reported in accordance with 2014 ASCO/CAP guidelines [35]. Results were reported as equivocal HER2 if there was weak to moderate, incomplete membranous staining in $>10 \%$ of cells or if FISH showed a HER2/CEP17 ratio $<2$, or if the HER2 copy number was $\geq 4$ and $<6$. HER2 results were negative if the immunohistochemistry or FISH assays fell below the thresholds for interpretation as equivocal. All assays were performed on pretreatment core biopsy samples.

\section{Pathology assessment}

Pathologic response was assessed by applying the MillerPayne grading criteria to definitive surgery specimens in comparison with initial core biopsies (Table 1). Two breast pathologists (AR and $\mathrm{PH}$ ) individually evaluated cases from their respective hospitals and additional cases from the third hospital. The Miller-Payne system [36] divides pathologic response into five grades based on a comparison of tumor cellularity between the pretreatment core biopsy and the definitive surgical specimen. Grade 1 indicates no change or some minor alteration in individual malignant cells but no reduction in overall cellularity; this is a pathological nonresponse (pNR). Grade 2 indicates a minor loss of tumor cells (up to $30 \%$ ) but with overall cellularity still high; this is a partial pathologic response (pPR). Grade 3 indicates an estimated $30-90 \%$ reduction in tumor cells (pPR). Grade 4 indicates a marked disappearance of tumor cells (>90\%), with only small clusters or widely dispersed individual cells remaining (almost pCR). Grade 5 indicates that no malignant cells are identifiable in sections from the tumor bed (pCR). Grade 5 may show that necrosis, granulation tissue, histiocytes, and vascular fibroelastotic stroma remains, often containing macrophages. Residual ductal carcinoma in situ (DCIS) is acceptable for MillerPayne grade 5.

\section{US and NIR system and imaging}

Ultrasound examinations were performed using a commercial ultrasound system (Phillips IU22 or GE Logiq 5) at the corresponding hospital. Three NIR systems with identical designs were used at the three hospitals, and the details have been given previously [24]. Briefly, the NIR/US probe consists of the commercial US transducer located centrally, with source and detector light guides (optical fibers) distributed around the periphery of the NIR/US probe. Four laser diodes of $740 \mathrm{~nm}, 780 \mathrm{~nm}$, $808 \mathrm{~nm}$, and $830 \mathrm{~nm}$ optical wavelengths were sequentially switched to nine positions on the probe, while the reflected light was coupled by the light guides to 14 parallel detectors. The entire NIR data acquisition interval was less than $5 \mathrm{~s}$. For each patient, US images and optical measurements were acquired simultaneously in the cancer region and a normal region within the contralateral breast in the same quadrant as the cancer. At each cancer and normal region, multiple datasets were acquired. The optical data acquired from the normal contralateral breast was used as a reference for calculating the background optical absorption and reduced scattering coefficients that were used in the image reconstruction of the lesions.

Details of the optical imaging reconstruction algorithm with experimental validation have been described elsewhere [37]. Briefly, the NIR reconstruction takes advantages of the ultrasound localization of lesions to segment the imaging volume into a region of interest (ROI) and background nonlesion regions. Since the spatial 
resolution of diffused light is poorer than that of US, the ROI is chosen to be at least two to three times larger than the tumor size measured by coregistered US in $x$ to $y$ dimensions. In addition, because the depth localization of diffused light is very poor, a tighter ROI in the depth dimension is set by using coregistered US. For each patient, the same size of ROI as that obtained from the pretreatment US is used for processing all datasets obtained at different treatment cycles. Therefore, the changes in tumor size seen by US during treatment have no major effect on the NIR image reconstruction. Among the 22 patients, two patients had initially palpable tumors with ill-defined and heterogeneous pretreatment ultrasound images. For these patients, tumor sizes estimated from pretreatment MRI measurements were used to assist in determining the US ROI in the $x$ to $y$ dimensions. The ROI in the depth dimension was typically set from the top border of the ill-defined tissue pattern to the chest wall as seen by US.

The optical absorption distribution at each wavelength was reconstructed and the tHb concentration, oxygenated hemoglobin (oxyHb) concentration, and deoxygenated hemoglobin (deoxyHb) concentration maps were computed from absorption maps at the four wavelengths [38]. The maximum values of $\mathrm{tHb}$, oxyHb, and deoxyHb were measured for each set of hemoglobin maps. For each patient imaged at each time point, an average maximum that was obtained from 5 to 10 quality NIR images at the tumor location was used to characterize the tumor. Data with patient motion as evaluated by using two coregistered US images before and after each NIR measurement were excluded from averaging. To assess the response of each patient, the tHb obtained before treatment was taken as the baseline and the percentage normalized to the baseline (\%tHb) was used to quantitatively evaluate the remaining tumor vascular fraction during chemotherapy.

\section{MRI and US imaging and measurements}

Nineteen patients had well-defined tumors visible by US. Tumor sizes were measured by US technologists under direct supervision of attending radiologists. The percentage ratio (\%US) of the largest dimension of each posttreatment measurement over the largest dimension of pretreatment measurement was used to evaluate the morphological change during NAC. One patient had a much larger tumor size than the US transducer, and the baseline US measurement was not accurate. The initial tumor mass of this patient measured by MRI was 6 . $9 \mathrm{~cm}$. US scans performed from both medial lateral and cranial-caudal directions using the 5-cm US transducer were used to estimate the approximate mass center. Then the combined probe of $10-\mathrm{cm}$ diameter was placed at the estimated mass center for NIR data acquisition.
This procedure had minimal effect on NIR reconstruction. For the two patients with initially palpable but illdefined and heterogeneous US images, the tumor location at each measurement was tracked using previous US images as references. The tumor clock position, distance of the tumor from the nipple, and depth below the skin were documented for each case. Additionally, the tumor posterior shadowing and surrounding tissue structures, as well as the metal clip position, were also reviewed and used to help identify the tumor for each subsequent measurement. If an MRI was ordered for clinical reasons, the MRI measurements were obtained from the medical records of the patients.

\section{Prediction models}

We have previously developed a logistic regression model to predict the NAC response of a patient using pretreatment tumor clinicopathologic variables, tumor subtype, and baseline tHb values [21]. Briefly, logistic regression is a statistical modeling approach that can be used to describe the relationship of several predictor variables $\mathrm{X}_{1}, \mathrm{X}_{2} \ldots \mathrm{X}_{\mathrm{k}}$ to a dichotomous response variable $\mathrm{Y}$, where $\mathrm{Y}$ is coded 1 (responder) or 0 (nonresponder) for its two possible categories [39]. The model can be written in the form of the conditional probability of the occurrence of one of the two possible outcomes of $\mathrm{Y}$, as follows:

$$
\begin{aligned}
\operatorname{pr}(Y=1 \mid \mathrm{X} 1, \mathrm{X} 2, \ldots \mathrm{Xk}) \\
=\frac{1}{1+\exp \left(-\left(\beta 0+\sum_{n=1}^{k} \beta n X n\right)\right)}
\end{aligned}
$$

The estimated outputs (probability) for each set of predictor variables range from 0 to 1 . Given the data on $\mathrm{Y}, \mathrm{X}_{1}, \ldots \mathrm{X}_{\mathrm{k}}$, the unknown parameters $\beta \mathrm{n}, \mathrm{n}=0,1, \ldots, \mathrm{k}$, $n=0,1, \ldots \mathrm{k}$ can be estimated using the maximum likelihood method.

In this study, we used the data from 32 patients obtained from an earlier study as a training set [20] to estimate a total of four groups of logistic models, and validated these models using the new dataset reported in this study as a testing dataset. The earlier data obtained from 2008 to 2011 were acquired from almost identical NIR systems with the same data processing and image reconstruction procedures as reported in this study.

To validate that the early data and new data are generated from the same population, we have introduced a dummy variable $X_{k+1}$ in Eq. 1 which is coded as 0 for early data and 1 as new data [40,41]. We have estimated the model with this dummy variable along with the eight predictor variables using the combined datasets. The estimate on $\beta_{k+1}(P=0.214)$ is statistically insignificant. 
Therefore, we can assume both datasets come from the same patient population.

The Matlab (version 2016a) logistic regression function glmfit was used to estimate the coefficients $\beta_{n} \beta n, n$ $=0,1, \ldots, k$, and glmval was used to calculate the receiver operating characteristic (ROC) with these coefficients for the training set. The same coefficients obtained from the training set were used to predict the response for the testing set.

\section{Evaluation of prediction models}

We also assessed the overall performance of the prediction models through the ROC curves and the area under the curves (AUCs) for each pair of training and testing sets of each prediction model. The early data used for training had $20 \mathrm{ER}^{+} / \mathrm{HER} 2^{-}$patients, six TN patients, five $\mathrm{HER} 2^{+}$patients, and one $\mathrm{ER}^{-} / \mathrm{PR}^{+} / \mathrm{HER} 2^{-}$patient [20]. Similar to the new patient cohort, $\mathrm{ER}^{+} / \mathrm{HER}^{-}(n=$ $14)$ and TN $(n=6)$ tumors were treated with ACT every 2 weeks for eight cycles. Six $\mathrm{ER}^{+} / \mathrm{HER} 2^{-}$tumors and one $\mathrm{ER}^{-} / \mathrm{PR}^{+} / \mathrm{HER} 2^{-}$tumor were treated with ACT $(n=3)$ or TC $(n=4)$ every 3 weeks for six cycles. HER2 ${ }^{+}$ tumors $(n=5)$ were treated with trastuzumab and docetaxel or paclitaxel with or without carboplatin (TPT) every 3 weeks for six cycles.

Since the early data did not contain any patients treated with dual HER2 blockade, we have randomly selected six HER $2^{+}$patients treated with this regimen from the current study and added these six datasets to the training data. For each random selection, the total of patients in training was 38 (32 from the early data and 6 from this study) and testing was 16 from this study. A total of 6 out of 11 random selections result in 462 combinations of paired training and testing datasets for each group of predictors, and the mean of 462 AUC values was used to evaluate the training and testing results of each prediction model. Each pair of training and testing ROCs was generated using a threshold of 0.5 . This was used to separate responders $(>0.5)$ and nonresponders $(\leq 0.5)$ for each prediction model output. We also used the 462 AUC values to construct the $95 \%$ confidence interval (CI) for the mean AUC for each model using a binomial formula. These confidence intervals can provide summary information on comparisons of the different models in terms of their AUC values. For example, if model I has a higher mean AUC than model II, and if their corresponding confidence intervals do not overlap, then this is an indication that model I may have a higher prediction power than the model II in terms of the AUC criterion. However, this interpretation should be understood with the caveat that the 462 values are not true random samples.

\section{Selection of predictors}

To select the independent predictors, Spearman's rho was evaluated between each predictor and Miller-Payne grade and between each pair of predictors. Spearman's rho is more appropriate for assessing the relationship for both continuous and discrete variables. Note that both training and testing data were combined to assess the predictors and the Spearman's rhos reported in this section are from the entire cohort of both earlier and new data (Table 2). To compute rho, the tumor HER2, ER, and TN status were coded as: 1 for TN and 0 for otherwise; 1 for $\mathrm{HER}^{+}$and 0 for HER2 ${ }^{-}$; and 0 for $\mathrm{ER}^{+}$and 1 for $E^{-}$. Note that 1 presents increased probability of achieving $\mathrm{pCR}$ and 0 otherwise.

Both HER2 and ER are highly correlated with MillerPayne grade (rho $=0.45, P<0.001$; rho $=0.29, P=0.035$ ) and are independent of each other (rho $=0.01, P=0$. 928); thus, they were selected as predictors. TN tumors did not have a significant correlation with Miller-Payne grade (rho $=0.124, P=0.362$ ). However, TN was selected as a predictor because it is used clinically to characterize this group of patients. Among tumor pathological parameters, NS is a traditional pathological variable used by oncologists to predict response. NS is highly correlated with mitotic counts (rho $=0.82, P<0.001$ ). Thus, only NS was selected as an independent pathological predictor and used in each HER2, ER, and TN subtypes to predict response.Baseline $\mathrm{tHb}$ and \% $\mathrm{tHb}$ changes measured during first three treatment cycles are highly correlated with Miller-Payne grade ( see Table 2) and were selected to assess the optimal time window to predict response.

\section{Statistical analysis}

A two-sample two-sided $t$ test was used to calculate the statistical significance for comparisons between responder

Table 2 Spearman's rho correlation coefficient and $P$ value between Miller-Payne grade and tumor pathological variables (MC, NS), tumor subtype (HER2, ER, TN), tHb, and \%tHb measured at the end of cycles 1 to 3

\begin{tabular}{llllllllll}
\hline & NS & MC & HER2 & ER & TN & tHb & \%tHb cycle 1 & \%tHb cycle 2 & \%tHb cycle 3 \\
\hline rho & 0.42 & 0.45 & 0.45 & 0.29 & 0.12 & 0.48 & 0.50 & 0.52 & 0.69 \\
$P$ & $P=0.001$ & $P<0.001$ & $P<0.001$ & $P=0.035$ & $P=0.364$ & $P<0.001$ & $P<0.001$ & $P<0.001$ & $P<0.001$ \\
\hline
\end{tabular}

Data are from [20] and this study ( $n=54$ patients)

ER estrogen receptor, HER2 human epidermal growth factor 2, MC mitotic count, NS Nottingham score, $t H b$ total hemoglobin, $T N$ triple negative 
groups, and a difference with a $P$ value of 0.05 was considered significant. The $t$ test was also used to test the difference between AUCs of different prediction models when their 95\% CIs overlapped. Minitab 17 software (Minitab, State College, PA) was used for statistical calculations.

\section{Results}

There were nine Miller-Payne grade 1-3 tumors and 13 grade $4-5$ tumors. For grade $4-5$ tumors, the pretreatment mean maximum $\mathrm{tHb}$ was $84.8 \pm 11.3 \mu \mathrm{mol} / \mathrm{L}$ (mean \pm standard deviation), whereas for grade 1-3 tumors the pretreatment mean maximum tHb was $67.9 \pm$ $16.2 \mu \mathrm{mol} / \mathrm{L}(P=0.018)$. The mean difference of the maximum $\mathrm{tHb}$ was $16.9 \mu \mathrm{mol} / \mathrm{L}$ and the $95 \% \mathrm{CI}$ was 3 . $5-3.04 \mu \mathrm{mol} / \mathrm{L}$. However, no statistical significance was found at day 7 and at the end of treatment cycles 1, 2, and 3 as the mean $\mathrm{tHb}$ level was reduced in grade 4-5 tumors but the mean level did not change in grade $1-3$ tumors (Fig. 1).

The pretreatment oxyHb was not significantly different between grade $4-5$ and grade $1-3$ tumors $(P=0.083)$; however, the deoxyHb difference was significant $(P=0$. 028) (Fig. 2). In subsequent measurements, no significant difference was observed in either oxyHb or deoxyHb.

The \% $\mathrm{tHb}$ (percentage fraction from baseline) was calculated and the results for the two groups are given in Fig. 3. No statistical significance between the two groups was found at day $7(P=0.238)$. Statistical significance was achieved at the end of cycle 1. For Miller-Payne grade $1-3$ tumors $\%$ tHb was $102 \pm 12 \%$, whereas for grade $4-5$ tumors $\% \mathrm{tHb}$ was $72 \pm 22 \%$. The mean difference was $30 \%(P=0.001)$, and the $95 \% \mathrm{CI}$ was $14.6-45$.
$0 \%$. The significance remained high at the end of cycles 2 and 3 , with mean differences of $26 \%(P=0.018)$ and 95\% CI 5.1-46.4\%, and 25\% $(P=0.012)$ and 95\% CI 6.4$43.7 \%$, respectively.

In this new cohort, Spearman's rhos calculated between Miller-Payne grade and pretreatment $\mathrm{tHb}$, oxyHb, and deoxyHb, as well as \%tHb measured at different cycles, reveal that the pretreatment maximum $\mathrm{tHb}$ and maximum deoxyHb have achieved statistical significance $(P=0.049$ and 0.030 ; Table 3$)$. The $\% \mathrm{tHb}$ values measured at the end of cycles 1,2 , and 3 are highly predictive $(P=0.002, P=0.006$, and $P=0.048$, respectively), while \% $\mathrm{tHb}$ measured at day 7 , the end of cycle 5 , and before operation are not predictive $(P=0.321, P=0.321$, and $P=0.202$, respectively).

There is no correlation between the pretreatment tumor size measured by MRI $(n=18, P=0.150)$ and US $(n=19, P=0.152)$ and the Miller-Payne grade.

An example of a pCR is shown in an HER2-positive tumor (Fig. 4) in a 51-year-old woman with a high-grade invasive ductal carcinoma treated with TPT every 3 weeks for six cycles. US images obtained at pretreatment, day 7 , at the completion of cycle 1 , and before surgery are shown in the left panel. The tumor was well defined and seen by US before treatment and at day 7 , was barely visible at the completion of cycle 1 , and was not visible at the completion of cycles 2 (data not shown) and 3 (data not shown), and before surgery. $\mathrm{tHb}$ concentration maps obtained at the corresponding time points are shown in the right panel. The tHb reduced from $85.8 \mu \mathrm{mol} / \mathrm{L}$ measured before treatment to 69.4 , 36.3 , and $21.8 \mu \mathrm{mol} / \mathrm{L}$ measured at day 7 , before the completion of cycle 1 , and before surgery, respectively. $\mathrm{tHb}$ measured at the completion of cycles 2 and 3 were

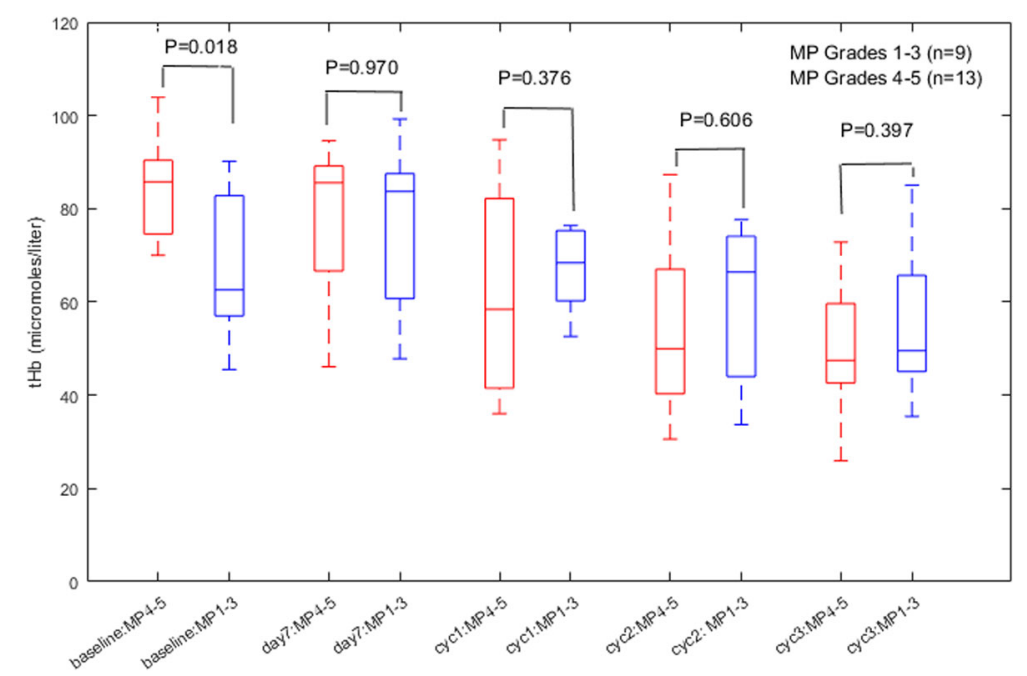

Fig. 1 Box plot of mean maximum total hemoglobin (tHb; $\mu$ mol/L) of two responder groups of Miller-Payne (MP) 4-5 and MP 1-3 measured at baseline, day 7, and at the end of cycles (cyc) 1, 2, and 3 of NAC 


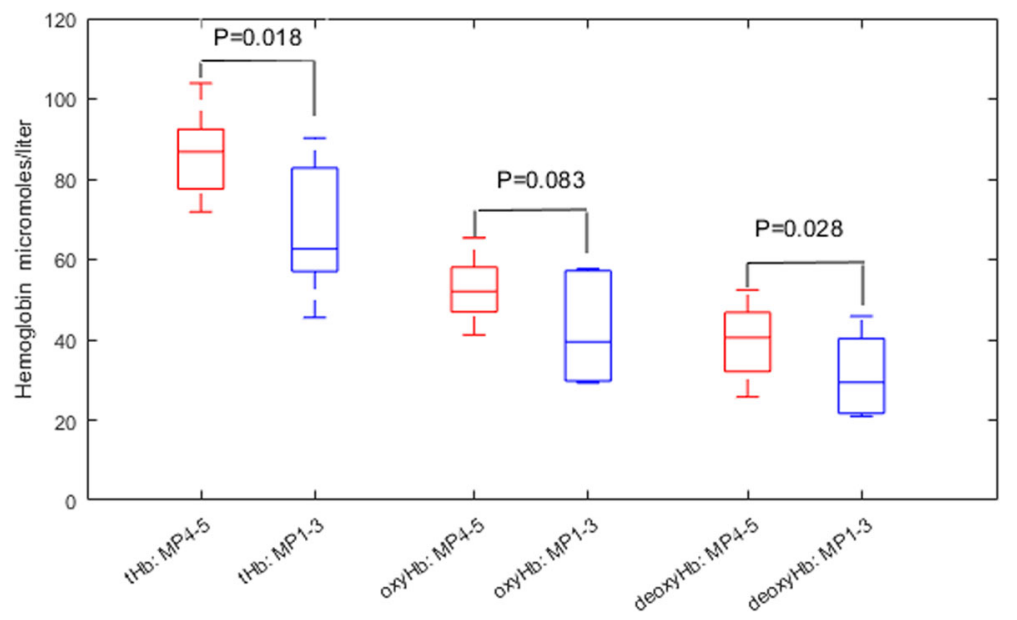

Fig. 2 Box plot of pretreatment maximum total hemoglobin ( $\mathrm{tHb}$ ), oxygenated hemoglobin (oxyHb), and deoxygenated hemoglobin (deoxyHb) $(\mu \mathrm{mol} / \mathrm{L}$ ) of two responder groups. MP, Miller-Payne

41.8 and $28.7 \mu \mathrm{mol} / \mathrm{L}$, respectively (data not shown). A dramatic tHb reduction occurred at the end of cycle 1 $(\% \mathrm{tHb}=42 \%)$. This patient had a complete pathologic response with no residual tumor (Miller-Payne grade 5).

An example of a partial responder is shown in a highgrade ER-positive/HER2-negative tumor (Fig. 5). A 72year-old woman had a locally recurrent invasive ductal carcinoma. She was treated with TC every 3 weeks for four cycles before surgery. US images at the four time points pretreatment, day 7 , at the completion of cycle 1 , and before surgery are shown in the left panel. The tumor was ill-defined with unclear boundary seen by US. tHb concentration maps obtained at the corresponding time points are shown in the right panel. tHbs of 90.2, 99.3, 108.8, 105.0 (data not shown), 85.0 (data not shown), and $69.0 \mu \mathrm{mol} / \mathrm{L}$ were measured at pretreatment, day 7 , at the completions of cycles 1 to 3 , and before surgery, respectively. The patient had a partial response with a residual invasive carcinoma of $2.8 \mathrm{~cm}$ (Miller-Payne grade 3).

Based on tumor biomarkers and hemoglobin measurements, predictors are grouped into four categories:1) HER2 status with hemoglobin predictors; 2) ER with hemoglobin predictors; 3) TN with hemoglobin predictors; and 4) hemoglobin predictors (see Table 4). ROCs of validation or testing of these four groups are given in Fig. 6 and Table 4. Note that predictor groups that achieve higher training AUCs do not necessarily

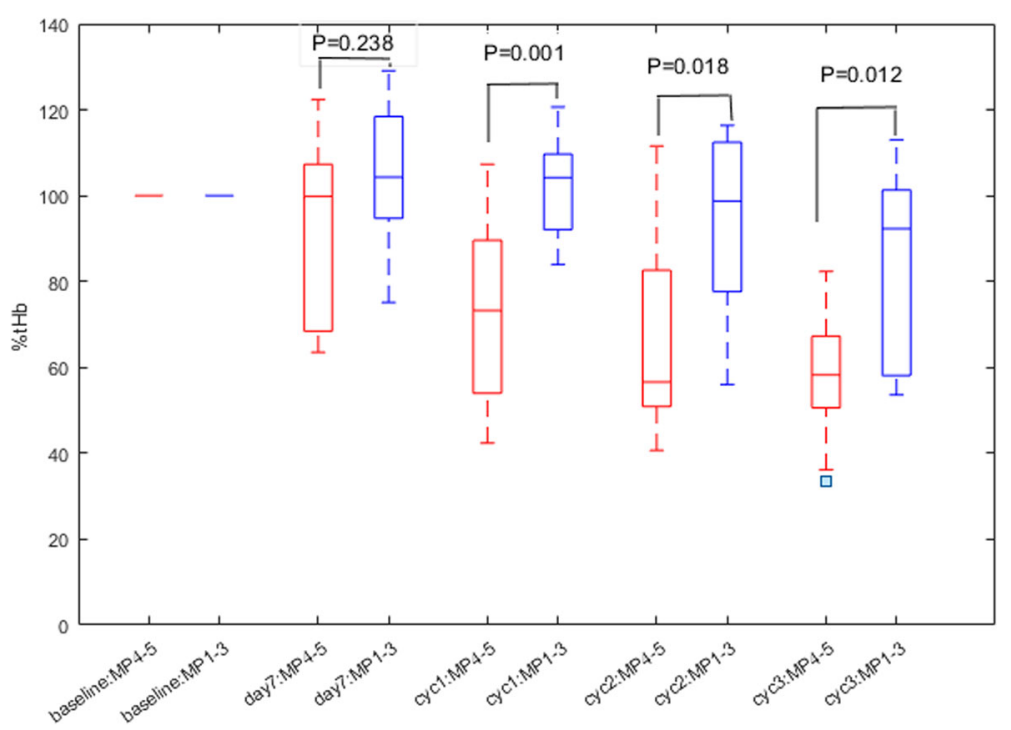

Fig. 3 Box plot of percent total hemoglobin (\%tHb) of two responder groups of Miller-Payne (MP) 4-5 and MP 1-3 measured at baseline, day 7, and at the end of cycles (cyc) 1, 2, and 3 of NAC 
Table 3 Spearman's rho correlation coefficient and $P$ value between Miller-Payne grade and pretreatment $\mathrm{tHb}$ (maximum), oxyHb (maximum), deoxyHb (maximum), \%tHb at day 7, and at the end of cycles 1, 2, 3, and 5, and before surgery

\begin{tabular}{|c|c|c|c|c|c|c|c|c|c|}
\hline & $\mathrm{tHb}(\max )$ & $\mathrm{oxyHb}$ (max) & deoxyHb (max) & $\% \mathrm{tHb}$ day 7 & $\% \mathrm{tHb}$ cycle 1 & $\% \mathrm{tHb}$ cycle 2 & \%tHb cycle 3 & \%tHb Cycle 5 & $\%$ thb before surgery \\
\hline rho & 0.43 & 0.28 & 0.46 & 0.26 & 0.61 & 0.57 & 0.43 & 0.26 & 0.29 \\
\hline$P$ & $P=0.049$ & $P=0.205$ & $P=0.030$ & $P=0.321$ & $P=0.002$ & $P=0.006$ & $P=0.048$ & $P=0.321$ & $P=0.202$ \\
\hline
\end{tabular}

Data are from the new cohort ( $n=22$ patients)

deoxy $\mathrm{Hb}$ deoxygenated hemoglobin, oxyHb oxygenated hemoglobin, $t H b$ total hemoglobin
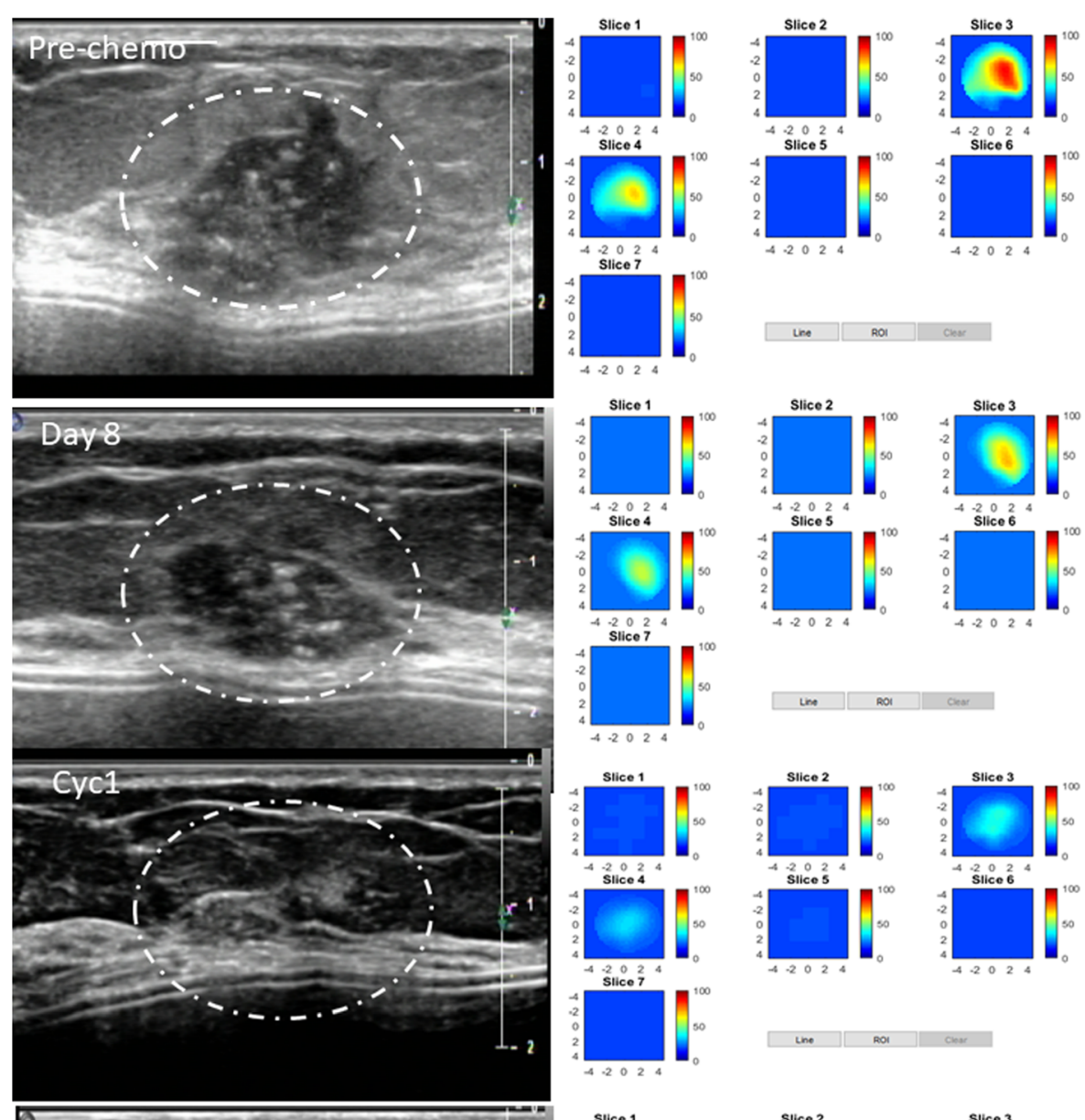

\begin{tabular}{|l|l|l|}
\hline Ino & ROI & Cles \\
\hline
\end{tabular}

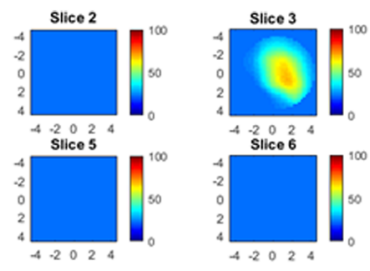

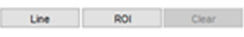
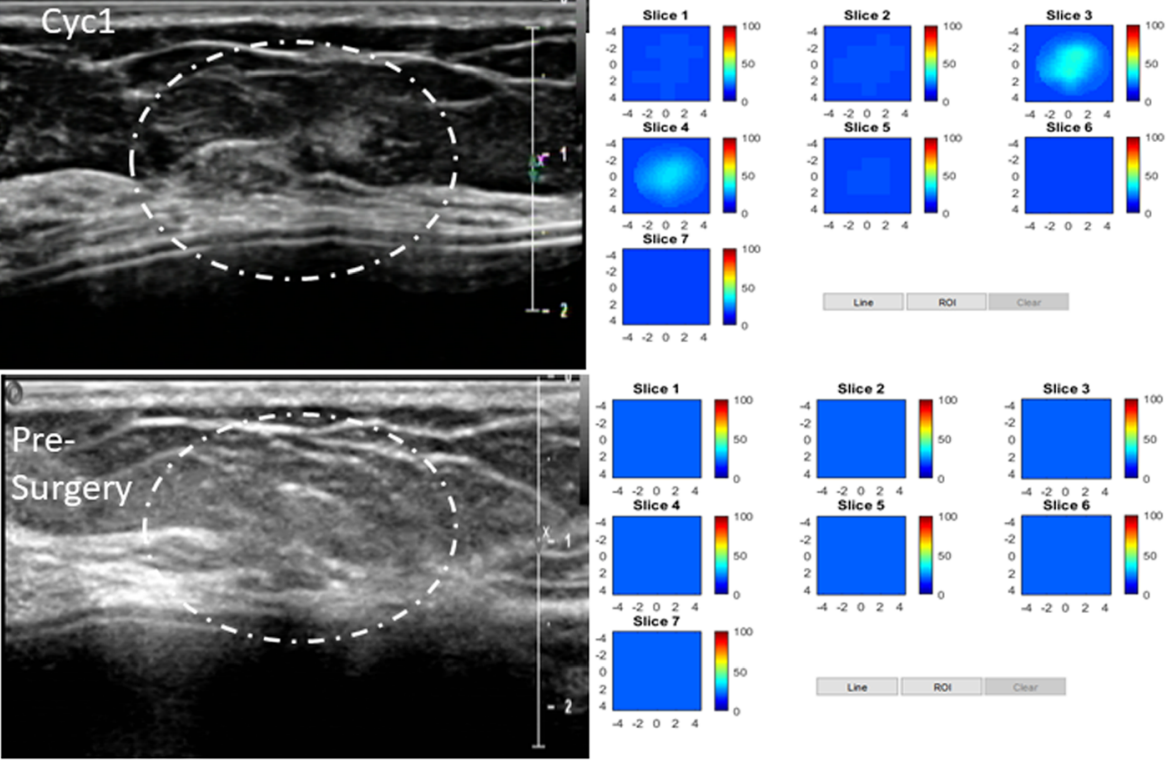

Fig. 4 pCR in an HER2-positive tumor in a 51-year-old woman with a high-grade invasive ductal carcinoma treated with TPT every 3 weeks for six cycles. Left panel: US images obtained at pretreatment, at day 7, at the completion of cycle 1, and before surgery. Right panel: tHb concentration maps obtained at the corresponding time points. Each map shows seven subimages marked as slice 1 to 7 , and each subimage shows spatial $x$ and $y$ distribution of $\mathrm{tHb}$ concentration reconstructed from $0.5 \mathrm{~cm}$ to $3.5 \mathrm{~cm}$ below the skin surface. The depth spacing between the subimages in depth is $0.5 \mathrm{~cm}$. The color bar is tHb in micromoles per liter. This patient had a complete pathologic response with no residual tumor, MillerPayne grade 5 

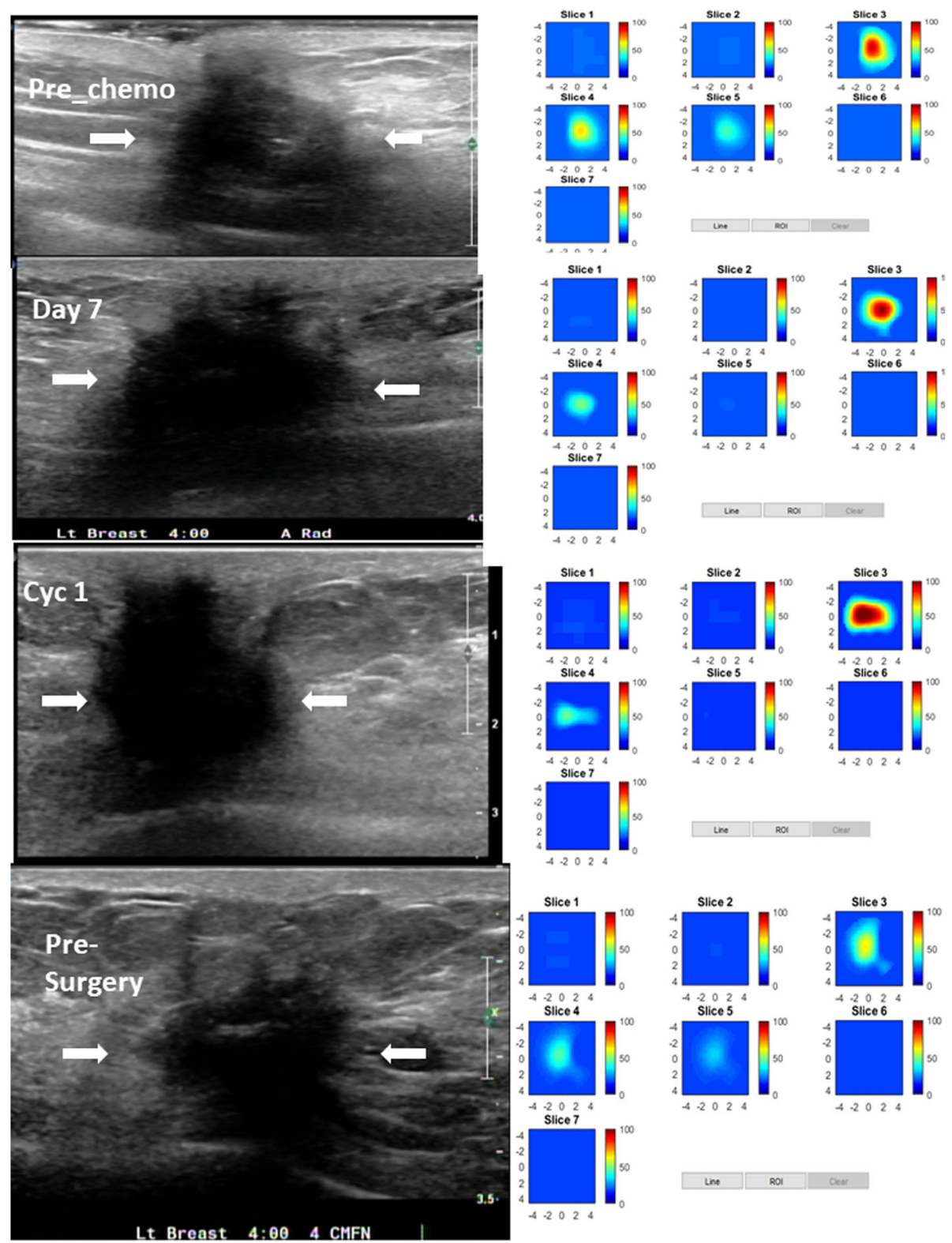

Fig. 5 Partial response in a high-grade ER-positive/HER2-negative tumor in a 72-year-old woman with a locally recurrent invasive ductal carcinoma. She was treated with cyclophosphamide and docetaxel every 3 weeks for four cycles before surgery. Left panel: US images at four time points of pretreatment, at day 7, at the completion of cycle (Сyc) 1, and before surgery. The tumor was ill-defined with an unclear boundary seen by US. Right panel: tHb concentration maps obtained at the corresponding time points. The patient had a partial response with residual invasive carcinoma of $2.8 \mathrm{~cm}$, Miller-Payne grade 3

translate into higher testing AUCs, and higher AUCs of testing data are used to compare prediction models.

For HER2 group 1, HER2 used alone can achieve an AUC of 0.78 (95\% CI 0.74-0.81). When HER2 and $\mathrm{tHb}$ are used together, the AUC reached 0.88 (95\% CI 0.85-0.91). The addition of ER or NS essentially produces similar AUCs of 0.87 (95\% CI 0.84-0.90) and 0. 85 (0.82-0.88). However, the statistical significance of HER2 and tHb is higher than HER2, tHb, and ER ( $P$ $=0.016)$ and HER2, tHb, and NS $(P<0.001)$. This suggests that HER2 and tHb are the best pretreatment predictors regardless of ER or NS. The highest AUC of 0.96 (95\% CI 0.95-0.98) is achieved when $\% \mathrm{tHb}$ measured at the end of cycle 1 is added to HER2 and tHb. This was further modestly improved to 0.97 (95\% CI 0.96-0.99) when \%tHb measured at end of cycles 1 and 2 are used together with HER2 and $\mathrm{tHb}$. Thus, the optimal time window with an accurate prediction of pathologic response in the HER2 subtype is at the end of cycle 2 . 
Table 4 Four groups of logistic regression models based on tumor subtype and hemoglobin parameters, AUC of training data, and AUC of testing data

\begin{tabular}{|c|c|c|}
\hline Tumor subtypes & Training AUC (95\% Cl) & Testing AUC $(95 \%$ \\
\hline \multicolumn{3}{|l|}{ Group 1 (HER2 subtype) } \\
\hline Her2 & $0.71(0.66-0.75)$ & $0.78(0.74-0.81)$ \\
\hline $\mathrm{Her} 2, \mathrm{tHb}$ & $0.88(0.85-0.91)$ & $0.88(0.85-0.91)$ \\
\hline Her2, tHb, ER & $0.91(0.89-0 / 94)$ & $0.87(0.84-0.90)$ \\
\hline Her2, tHb, NS & $0.91(0.88-0.94)$ & $0.85(0.82-0.88)$ \\
\hline Her2, tHb, \%tHb_cyc1 & $0.89(0.87-0.92)$ & $0.96(0.95-0.98)$ \\
\hline Her2, tHb, \%tHb_cyc2 & $0.89(0.86-0.91)$ & $0.94(0.92-0.96)$ \\
\hline Her2, tHb, \%tHb_cyc3 & $0.96(0.94-0 / 97)$ & $0.89(0.86-0.92)$ \\
\hline Her2, tHb, \%tHb_cyc1, \%tHb_cyc2 & $0.90(0.87-0.93)$ & $0.97(0.96-0.99)$ \\
\hline Her2, tHb, \%tHb_cyc1, \%tHb_cyc3 & $0.96(0.94-0.97)$ & $0.88(0.85-0.91)$ \\
\hline Her2, tHb, \%tHb_cyc2, \%tHb_cyc3 & $0.96(0.94-0.98)$ & $0.88(0.85-0.91)$ \\
\hline Her2, tHb, \%tHb_cyc1,\%tHb_cyc2, \%tHb_cyc3 & $0.96(0.94-0.98)$ & $0.88(0.86-0.92)$ \\
\hline \multicolumn{3}{|l|}{ Group 2 (ER subtype) } \\
\hline ER & $0.67(0.63-0.72)$ & $0.55(0.50-0.59)$ \\
\hline $\mathrm{ER}, \mathrm{tHb}$ & $0.81(0.77-0.85)$ & $0.69(0.64-0.73)$ \\
\hline $\mathrm{ER}, \mathrm{tHb}, \mathrm{NS}$ & $0.83(0.80-0.87)$ & $0.69(0.65-0.73)$ \\
\hline ER, tHb, \%tHb_cyc1 & $0.85(0.82-0.89)$ & $0.91(0.88-0.93)$ \\
\hline $\mathrm{ER}, \mathrm{tHb}, \% \mathrm{tHb} \_\mathrm{cyc} 2$ & $0.86(0.83-0.89)$ & $0.79(0.75-0.83)$ \\
\hline $\mathrm{ER}, \mathrm{tHb}, \% \mathrm{tHb} \_\mathrm{cyc} 3$ & $0.97(0.95-0.98)$ & $0.77(0.73-0.81)$ \\
\hline ER, tHb, \%tHb_cyc1, \%tHb_cyc2 & $0.88(0.85-0.91)$ & $0.86(0.83-0.89)$ \\
\hline ER, tHb, \%tHb_cyc1, \%tHb_cyc3 & $0.96(0.95-0.98)$ & $0.76(0.73-0.80)$ \\
\hline ER, tHb, \%tHb_cyc2, \%tHb_cyc3 & $0.97(0.95-0.98)$ & $0.77(0.73-0.81)$ \\
\hline \multicolumn{3}{|l|}{ Group 3 (TN subtype) } \\
\hline $\mathrm{TN}$ & $0.55(0.51-0.56)$ & $0.46(0.41-0.50)$ \\
\hline $\mathrm{TN}, \mathrm{tHb}$ & $0.77(0.74-0.81)$ & $0.72(0.68-0.76)$ \\
\hline $\mathrm{TN}, \mathrm{tHb}$, NS & $0.81(0.78-0.85)$ & $0.69(0.65-0.75)$ \\
\hline TN, tHb, \%tHb_cyc1 & $0.84(0.81-0.87)$ & $0.90(0.87-0.93)$ \\
\hline TN, tHb, \%tHb_cyc2 & $0.85(0.82-0.88)$ & $0.84(0.81-0.88)$ \\
\hline $\mathrm{TN}, \mathrm{tHb}, \% \mathrm{tHb} \_\mathrm{cyc3}$ & $0.96(0.94-0.98)$ & $0.76(0.72-0.80)$ \\
\hline TN, tHb, \%tHb_cyc1, \%tHb_cyc2 & $0.85(0.82-0.88)$ & $0.90(0.87-0.93)$ \\
\hline TN, tHb, \%tHb_cyc1,\%tHb_cyc3 & $0.96(0.94-0.98)$ & $0.75(0.71-0.79)$ \\
\hline TN, tHb, \%tHb_cyc2, \%tHb_cyc3 & $0.96(0.94-0.98)$ & $0.75(0.71-0.79)$ \\
\hline \multicolumn{3}{|l|}{ Group 4 (tHb and \%tHb, all patients) } \\
\hline $\mathrm{tHb}$ & $0.77(0.73-0.81)$ & $0.75(0.71-0.79)$ \\
\hline $\mathrm{tHb}, \% \mathrm{HHb}$ _cyc1 & $0.83(0.80-0.87)$ & $0.95(0.93-0.97)$ \\
\hline tHb, \%tHb_cyc2 & $0.84(0.80-0.86)$ & $0.87(0.84-0.90)$ \\
\hline tHb, \%tHb_cyc3 & $0.94(0.92-0.97)$ & $0.80(0.76-0.84)$ \\
\hline tHb, \%tHb_cyc1, \%tHb_cyc2 & $0.84(0.81-0.88)$ & $0.92(0.90-0.95)$ \\
\hline tHb, \%tHb_cyc1, \%tHb_cyc2, \%tHb_cyc3 & $0.94(0.92-0.96)$ & $0.80(0.76-0.84)$ \\
\hline \%tHb_cyc1 & $0.79(0.75-0.83)$ & $0.89(0.86-0.92)$ \\
\hline \%tHb_cyc2 & $0.83(0.80-0.86)$ & $0.81(0.78-0.85)$ \\
\hline$\% \mathrm{tHb} \_\mathrm{cyc3}$ & $0.94(0.92-0.96)$ & $0.82(0.78-0.85)$ \\
\hline \%tHb_cyc1, \%tHb_cyc2 & $0.82(0.78-0.87)$ & $0.87(0.83-0.90)$ \\
\hline
\end{tabular}


Table 4 Four groups of logistic regression models based on tumor subtype and hemoglobin parameters, AUC of training data, and AUC of testing data (Continued)

\begin{tabular}{lll}
\hline Tumor subtypes & Training AUC (95\% Cl) & Testing AUC (95\% Cl) \\
\hline \%tHb_cyc1, \%tHb_cyc2, \%tHb_cyc3 & $0.94(0.92-0.96)$ & $0.82(0.79-0.86)$ \\
\hline
\end{tabular}

Bold entries indicate the best set of predictors in each group

$A U C$ area under the curve, $C l$ confidence interval, $E R$ estrogen receptor, $H E R 2$ human epidermal growth factor receptor $2, N S$ Nottingham score, $t H b$ total hemoglobin, $T N$ triple negative

For ER group 2, ER used alone can achieve an AUC of 0.55 (95\% CI 0.50-0.59). ER and tHb achieve AUC of 0 . 69 (95\% CI 0.64-0.73), and ER, tHb, and NS achieve AUC of 0.69 (95\% CI $0.65-0.73)$. NS does not add any value to predicting the response of the ER patient group. The addition of $\% \mathrm{tHb}$ measured at the end of cycle 1 improves the AUC of ER and tHb to 0.91 (95\% CI 0.88$0.93)$. For TN group 3, TN used alone can achieve an AUC of 0.46 (95\% CI $0.40-0.50)$. TN and tHb achieve an AUC of 0.72 (95\% CI 0.68-0.76), and TN, tHb, and NS achieve an AUC of 0.69 (95\% CI 0.65-0.75). NS does not add any value to predicting the response of the TN patient group. The addition of \%tHb measured at cycle 1 improves the AUC to 0.90 (95\% CI 0.87-0.93). For both ER and TN subtypes, \%tHbs measured at the end of cycles 2 and 3 do not significantly improve prediction (Table 4). Thus, the optimal time window for assessing response in ER or TN subtypes is at the end of cycle 1.
For group 4, tHb used alone can achieve an AUC of 0. 75 (95\% CI $0.71-0.79$ ), and \%tHb cycle 1 used alone can achieve an AUC of 0.89 (95\% CI 0.86-0.92). tHb and \%tHb cycle 1 achieve an AUC of 0.95 (95\% CI 0.93-0. 97), which is significantly higher compared with the ER or TN subtype groups $(P<0.001)$. \%tHbs measured at the end of cycles 2 and 3 do not significantly improve prediction (Table 4). Therefore, for ER and TN subtypes, $\mathrm{tHb}$ and \% $\mathrm{tHb}$ are the best predictors and the early window for prediction is at the end of cycle 1.

The sensitivity, specificity, positive predictive value (PPV), and negative predictive values (NPV) of the best groups of predictors are shown in Table 5.

The \%US ratio, the largest dimensions of posttreatment US measurements normalized to the pretreatment, of grade 1-3 and 4-5 tumors were calculated for 19 patients with well-defined US images. For grade 1-3 tumors $(n=9)$, \%USs were $90.1 \pm 9.8 \%, 84.9 \pm 17.3 \%$,
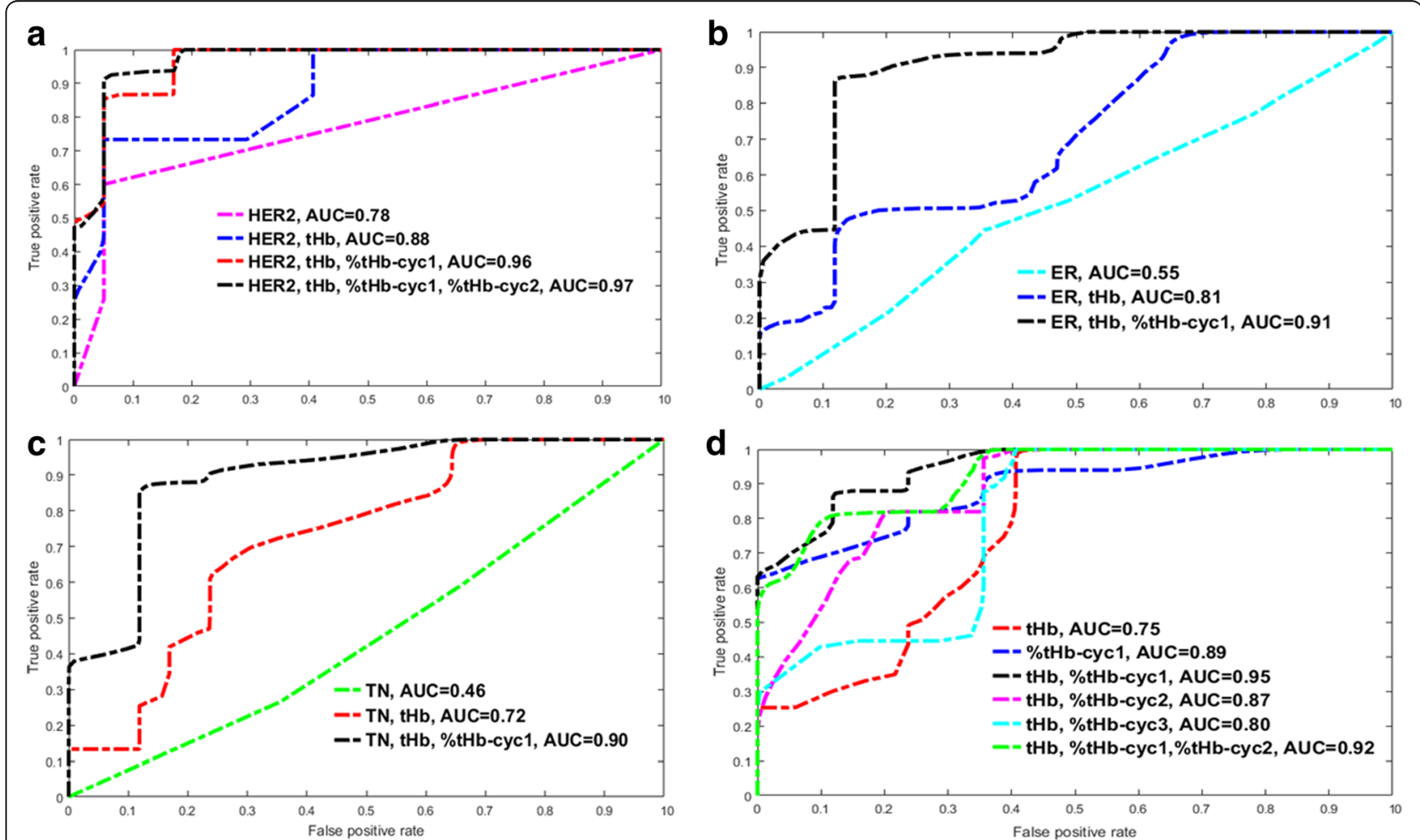

Fig. 6 ROC curves of testing data of four groups of prediction models based on a HER2 subtype and hemoglobin parameters, $\mathbf{b}$ ER subtype and hemoglobin parameters, c TN subtype and hemoglobin parameters, and $\mathbf{d}$ hemoglobin parameters 
Table 5 Sensitivity, specificity, PPV, NPV, and AUC of the best set of predictors based on tumor subtype and hemoglobin parameters of the testing data

\begin{tabular}{|c|c|c|c|c|c|}
\hline & Sensitivity & Specificity & PPV & NPV & AUC \\
\hline Her2, tHb, \%tHb_cyc1 & 73.7 & 94.9 & 92.4 & 84.8 & 0.96 \\
\hline Her, tHb, \%tHb_cyc1, \%tHb_cyc2 & 82.4 & 94.9 & 92.5 & 86.7 & 0.97 \\
\hline $\mathrm{ER}, \mathrm{tHb}, \% \mathrm{tHb} \_c y c 1$ & 81.8 & 88.1 & 85.8 & 85.1 & 0.90 \\
\hline TN, tHb, \%tHb_cyc1 & 82.0 & 88.1 & 85.9 & 85.1 & 0.90 \\
\hline $\mathrm{tHb}, \% \mathrm{tHb}$ _cyc1 & 83.8 & 88.1 & 86.1 & 86.5 & 0.95 \\
\hline
\end{tabular}

$A U C$ area under the curve, ER estrogen receptor, HER2 human epidermal growth factor receptor 2, NPV negative predictive value, $P P V$ positive predictive value, $t H b$ total hemoglobin, $T N$ triple negative

and $77.0 \pm 20 \%$ measured at end of cycles 1 to 3 , respectively, whereas for grade $4-5$ tumors $(n=11)$, \%USs were $61.2 \pm 18.0 \%, 52.3 \pm 15.6 \%$, and $42.8 \pm 13.7 \%$, respectively. Statistical significance was achieved at all cycles $(P<0.001, P<0.001$, and $P=0.001$, respectively).

\section{Discussion}

The clinical inclination to select patients for NAC who are more likely to be good responders accounts for the large number $(11 / 22,50 \%)$ of HER $2^{+}$tumors in this study, especially with the ability to utilize dual HER2 blockade in this setting. In the HER2 ${ }^{+}$group, there were Miller-Payne grade 4 and 5 responses in 91\% despite the large number of cases that coexpressed ER. This apparent anomaly of HER2/ER coexpression is partially explained by the relatively low-level ER expression in two of the eight $\mathrm{HER}^{+} / \mathrm{ER}^{+}$cases. The remaining patients were either $\mathrm{ER}^{+} / \mathrm{HER} 2^{-}$or $\mathrm{TN}$ and had Miller-Payne 4 or 5 responses of $17 \%$ and $40 \%$, respectively.

In our previous work, we dichotomized our comparison groups as pCR and near pCR (Miller-Payne grades 4-5) versus "other" (Miller-Payne grades 1-3). Our rationale for using these same comparison groups in this report is further supported as follows. In the original study by Ogston [36], the Miller-Payne 5 and 4 groups tended to track together with regard to 5-year diseasefree survival after NAC and surgery (85\% and 72\%) versus $66 \%, 60 \%$, and $55 \%$ for Miller-Payne 1-3, respectively. Later, Zhao et al. [42] while evaluating the MillerPayne system using a different dataset found very similar 5-year distant disease-free survival and local recurrencefree survival rates for Miller-Payne 4-5 versus MillerPayne 1-3. Finally, Symmans et al. [43] using the residual cancer burden (RCB) system and another separate dataset for evaluating tumor response after NAC also found that $\mathrm{pCR}$ and near-pCR had very similar survival curves after surgery (their categories RCB-0 and RCB-1 compared with RCB-2 (partial response) and RCB-3 (chemoresistant)).

In studies published to date, diffused optical tomography and diffused optical spectroscopy have demonstrated the potential for predicting breast cancer pathological response. Studies have shown that accurate predictions were made in the neoadjuvant setting by utilizing pretreatment hemoglobin levels or blood oxygen saturations $\left(\mathrm{SO}_{2}\right)[20,21,23,31]$, or by monitoring early changes of hemoglobin content and $\mathrm{SO}_{2}$ at 1 day or 1 week $[19,27]$, or after the first two cycles of NAC $[20,22,23]$. In this study, we have developed prediction models and have shown that the best pretreatment predictors are HER2 and tHb $(\mathrm{AUC}=0.88)$. The pretreatment predictors based on ER and $t \mathrm{Hb}$, and $\mathrm{TN}$ and $\mathrm{tHb}$ predict response with moderate AUC accuracies of AUC 0.69 and 0.72 , which are about the same as for the single predictor $\mathrm{tHb}(\mathrm{AUC}=0.75)$. For the HER2 subtype, the best window to accurately predict response is at the completion of the first two cycles of NAC. For ER or TN subtypes, the best window is at the completion of the first cycle of NAC and the best predictors are $\mathrm{tHb}$ and $\% \mathrm{tHb}$.

In our earlier study of 32 patients [21], the testing data obtained from cross-validations showed that the addition of the pretreatment $\mathrm{tHb}$ to pathological variables and biomarkers significantly improved the prediction (AUC 0.92 (95\%CI 79.4-99.8)) compared with using these variables alone (AUC 0.84 (95\% CI 57.2-99.0). The best pretreatment predictors of HER2, ER, and $\mathrm{tHb}$ reported in this study using the new cohort data as the testing set has achieved similar results of AUC 0.87 (95\% CI 0.84$0.90)$.

NIR/US measurements obtained at the end of the first three treatment cycles were used for development and validation of the prediction models. Chemotherapy treatments are delivered generally in specific cycles. These schedules are based on maximal tumor cell kill and allowance of recovery of normal tissues (http://chemocare. com/chemotherapy/what-is-chemotherapy/cancer-cellschemotherapy.aspx). Some cycles are given every 2 weeks and others every 3 weeks. Tumor responses by imaging studies occur after a specified number of treatment cycles, and not by specific times. Because treatments vary for HER2-positive disease compared with ER-positive or TN disease, the drugs utilized and schedules vary. The effects of the treatments are studied by 
equivalent treatment cycles. The goal was to give guideline-based treatments and to measure the maximal effect of each of these treatments before the next cycle is given. When combining all patients together, the measurements points were different in terms of weeks but not by cycles-they were consistent. The results show that pretreatment $\mathrm{tHb}$ and first cycle $\% \mathrm{tHb}$ can achieve an accuracy of AUC 0.96 in the HER2 subtype, and AUCs of 0.91 in ER and 0.90 in TN subtypes, and AUC of 0.95 regardless of subtype. Thus, it is the ultimate effect of the drug on the tumor vascularity that is being assessed by near infrared functional parameters.

Baseline tHb measures tumor angiogenesis and correlates with tumor aggressiveness as evaluated by Spearman's rhos with Nottingham score (rho $=0.355, P=0$. $007)$ in the combined training and testing data of 54 patients. Aggressive tumors have high proliferative rates and respond quickly to chemotherapy, as shown by $\mathrm{PET} / \mathrm{CT}$ which detects pretreatment and early changes in tumor metabolic activity after one or two cycles of NAC [44] and predicts pCR. As expected from log cellkill kinetics of cytotoxic drugs, a given dose kills a constant proportion of a tumor cell population rather than a constant number of cells (https://bajan.files.wordpress. com/2010/09/principles-of-cancer-chemotherapy.pdf).

Therefore, for chemosensitive tumors, there are more total cells killed in the first cycle of treatment and more tumor neovasculature damage that may cause a significant decrease in tumor hemoglobin measured by the NIR system. For HER2 ${ }^{+}$responders, the cycle $1 \% \mathrm{tHb}$ is lower (mean $\% \mathrm{tHb}=77 \%$ ) and more predictive than that of $\mathrm{ER}^{+} / \mathrm{HER} 2^{-}$and $\mathrm{TN}$ responders (mean \% $\mathrm{tHb}=84 \%$ ) because trastuzumab has been demonstrated to have a strong antiangiogenic effect [45].

Our study has substantial implications for the combined use of tumor subtypes and NIR-measured tumor hemoglobin content in predicting pathological response even before therapy has begun. If a decision is made to initiate therapy, modification of the treatment can be implemented as soon as cycles 1 and 2 are completed, allowing for personalized treatment. This ability will be of even greater value as our armamentarium of interventions increases and responses can more effectively tailor the agents selected.

In this new cohort of patients, $86 \%$ of the tumors were visible on US compared with $65 \%$ in our earlier study [20]. This difference could be due to a greater representation of HER2-positive tumors in which US has been shown to be more accurate in measuring tumor size [46]. Pretreatment US does not predict response. However, \%US of this cohort demonstrated statistical significance between responders and nonresponders at the early treatment cycles 1 to 3 , while the earlier study group [20] showed no statistical significance at the end of cycles 1 and $2(P=0.437$ and $P=0.172)$ between these two responder groups. Significance was achieved at the end of cycle 3. Additionally, earlier data showed no correlation between \%US measured at the end of cycles 1 to 3 and Miller-Payne grade ( $\mathrm{rho}=0.27, P=0.211$; rho $=0$. $25, P=0.257$; and rho $=0.36, P=0.106$ ), respectively. With more patient data, the \%US measure will be assessed on its role in predicting response.

There are some limitations to this study. First, all patients were referred for NAC after core biopsy, and hence the baseline NIR/US imaging was performed after the initial biopsy. Bruise or hematoma due to prior biopsy could have some effect on pretreatment NIR measurements. However, 21 patients had the pretreatment NIR measurements more than 10 days and one patient 7 days after biopsy. Based on the literature, the pretreatment NIR measurements 1 week after core biopsy were not affected by the biopsy [47]. However, another study followed a patient before and after biopsy and showed a $10 \%$ increase in diffuse optical spectroscopy (DOS)-measured deoxygenated hemoglobin after 9 days following biopsy [48]. Our study patient who imaged 7 days following biopsy had a pCR of Miller-Payne grade 5, and her cycle $1 \% \mathrm{tHb}$ was $58 \%$, or a reduction of $42 \%$. This level of \%tHb change is too large to be counted as a biopsy effect. Secondly, this new pool of 22 patients had $50 \% \mathrm{HER}^{+}$tumors and were treated with the dual HER2 blockade regimen which was not available in the training data obtained from 2008 to 2011 . We have randomly selected data from 6 out of 11 patients from this study and added these 6 patients' data to the training set to train the prediction models using all combinations. The testing data includes $73 \%$ from this study, with adequate samples of all subtypes. Thirdly, the training and testing datasets are still small and overfitting can occur when the training dataset is limited [21]. We have selected the minimal number of independent predictors for each prediction model, performed partial cross-validation, and used fairly high amounts $(16 / 54,30 \%)$ of the patient data for testing. The performances of the prediction models based on respective training and testing datasets are similar with no obvious pattern of higher AUC values for training data and much lower AUCs for testing data, which would be expected if there were problem of overfitting. With more patients recruited to the study, we will be able to establish a large database to validate prediction models with more input predictors.

The technical limitations of the US-guided NIR technique include the accuracy of the reconstructed optical absorption coefficients, the longitudinal repeatability of the measurements, and $\mathrm{SO}_{2}$ estimation. For a large high-contrast phantom of $3-5 \mathrm{~cm}$ in size, about $60-70 \%$ reconstruction accuracy in target absorption can be 
achieved [38]. Because the average pretreatment tumor sizes of the two responder groups were similar, any under-reconstruction should affect the light quantification of both groups similarly. Therefore, the comparison of pretreatment and early treatment hemoglobin levels between the two responder groups should be minimally affected. Additionally, because the same sized ROI obtained from the pretreatment US of each patient is used for reconstructions at all subsequent treatment cycles for the same patient, under-reconstruction should have a minimal effect on the \% $\mathrm{tHb}$, which is normalized to the pretreatment level. The longitudinal repeatability of the reconstructed phantom absorption coefficient is about $5-10 \%$, which is obtained by repeatedly imaging solid absorbers embedded in the same concentration of Intralipid over a 1- to 2-year period. This level of change is much smaller than the changes seen in patients who responded to treatments. Finally, $\mathrm{SO}_{2}$ estimated from DOS has been reported as a good pretreatment predictor [31]. However, $\mathrm{SO}_{2}$ distribution $=0 x y H b$ distribution/tHb distribution is not as robust as tHb, oxyHb, and deoxyHb when the tHb values reconstructed from tomography are lower in some voxels, especially when the tumor is large and distribution is heterogeneous.

\section{Conclusions}

In conclusion, our findings indicate that the breast tumor biomarkers (HER2, TN, and ER) combined with the pretreatment tumor total hemoglobin content are strong predictors of the response to NAC. The optimal treatment window to identify patients destined to have complete or near-complete responses is after the completion of the first two treatment cycles for HER2 tumors and the first treatment cycle for ER or TN tumors, when the assessment of total hemoglobin change is further predictive. This technology could be a valuable tool in personalizing treatments by response. These initial results remain to be validated with a larger trial of more patients.

\footnotetext{
Abbreviations

AUC: Area under the ROC curve; Cl: Confidence interval; CT: Computed tomography; deoxyHb: Deoxygenated hemoglobin; DOS: Diffuse optical spectroscopy; ER: Estrogen receptor; FISH: Fluorescence in situ hybridization; HER2: Human epidermal growth factor receptor 2; MRI: Magnetic resonance imaging; NAC: Neoadjuvant chemotherapy; NIR: Near infrared; NPV: Negative predictive value; NS: Nottingham histologic score; oxyHb: Oxygenated hemoglobin; pCR: Pathological complete response; PET: Positron emission tomography; PPV: Positive predictive value; PR: Progesterone receptor; RCB: Residual cancer burden; ROC: Receiver operating characteristic; ROI: Region of interest; $\mathrm{SO}_{2}$ : Oxygen saturation; $\mathrm{tHb}$ : Total hemoglobin; TN: triple negative; US: Ultrasound
}

\section{Acknowledgements}

The authors thank the clinical trial office of Hartford Hospital for helping with patient consenting and scheduling. The authors appreciate the help of Jadwiga Jerman, MD, of Waterbury Hospital, and Ali Quratulain of UCONN Cancer Center for patient consenting and scheduling.

\section{Funding}

The authors thank the funding support of this work from the National Institute of Health (R01EB002136) and Connecticut Bioscience Innovation Fund (CBIF) Award \#513.

\section{Availability of data and materials}

Data are available upon reasonable request.

\section{Authors' contributions}

QZ: designed and conducted all aspects of the ultrasound-guided tomography data acquisition, image reconstruction and data analysis, and contributed to the manuscript preparation and literature review. ST: recruited neoadjuvant chemotherapy patients at UConn Health Center, coordinated all aspects of the study at UCONN Health Center, and contributed to the manuscript preparation and literature review. SHK: coordinated all aspects of the clinical study at Waterbury Hospital and contributed to the manuscript preparation. PD: recruited neoadjuvant chemotherapy patients at Hartford Hospital and coordinated all aspects of the study at Hartford Hospital. AR: assessed and interpreted all histopathologic data of the breast tissue samples of patients recruited from Hartford Hospital and Waterbury Hospital, and contributed to the manuscript preparation and literature review. HV, FZ, and CX: contributed to the near-infrared system development, testing and calibration, and data acquisition. AM and MK: contributed to the US evaluations of the neoadjuvant chemotherapy patients and manuscript review. $\mathrm{PH}$ : assessed and interpreted all histopathologic data of the breast tissue samples of patients recruited from UConn Health Center and Waterbury Hospital. LW: contributed to the statistical analysis and manuscript preparation. KS: recruited neoadjuvant chemotherapy patients at Waterbury Hospital and coordinated oncological aspects of the study at Waterbury Hospital. All authors read and approved the final manuscript.

Ethics approval and consent to participate

The study protocol was approved by the institutional review boards of the University of Connecticut Health Center, Hartford Hospital and Waterbury Hospital and was HIPAA compliant. Written informed consent was obtained from all patients.

\section{Consent for publication}

All authors have read the manuscript and agreed with the submission.

\section{Competing interests}

QZ is the inventor of patents related to ultrasound-guided near-infrared tomography technologies and patents owned by the University of Connecticut. The remaining authors declare that they have no competing interests.

\section{Publisher's Note}

Springer Nature remains neutral with regard to jurisdictional claims in published maps and institutional affiliations.

\section{Author details}

${ }^{1}$ Biomedical Engineering and Radiology, Washington University in St Louis, One Brookings Drive, Mail Box 1097, Whitaker Hall 300D, St. Louis, MO 63130, USA. ${ }^{2}$ University of Connecticut Health Center, Farmington, CT 06030, USA. ${ }^{3}$ Waterbury Hospital, Waterbury, CT 6708, USA. ${ }^{4}$ Hartford Hospital, Hartford, CT 06102, USA. ${ }^{5}$ University of Connecticut, Storrs, CT 06269, USA. ${ }^{6}$ New York City College of Technology, City University of New York (CUNY), New York, USA. ${ }^{7}$ Department of Statistics, University of Manitoba, 186 Dysart Road, Winnipeg, Manitoba R3T 2N2, Canada.

Received: 31 October 2017 Accepted: 30 April 2018

Published online: 14 June 2018

\section{References}

1. Kaufmann M, von Minckwitz G, Mamounas EP, Cameron D, Carey LA, Cristofanilli M, Denkert C, Eiermann W, Gnant M, Harris JR, Karn T, Liedtke C, Mauri D, Rouzier R, Ruckhaeberle E, Semiglazov V, Symmans WF, Tutt A, Pusztai L. Recommendations from an international consensus conference on the current status and future of neoadjuvant systemic therapy in primary breast cancer. Ann Surg Oncol. 2012;19(5):1508-16. 
2. Cortazar P, Geyer CE Jr. Pathological complete response in neoadjuvant treatment of breast cancer. Ann Surg Oncol. 2015;22(5):1441-6. https://doi. org/10.1245/s10434-015-4404-8.

3. Hennessy BT, Hortobagyi GN, Rouzier R, Kuerer H, Sneige N, Buzdar AU, Kau SW Fornage B, Sahin A, Broglio K, Singletary SE, Valero V. Outcome after pathologic complete eradication of cytologically proven breast cancer axillary node metastases following primary chemotherapy. J Clin Oncol. 2005;23(36):9304-11.

4. Gianni L, Eiermann W, Semiglazov V, Manikhas A, Lluch A, Tjulandin S, Zambetti M, Vazquez F, Byakhow M, Lichinitser M, Climent MA, Ciruelos E, Ojeda B, Mansutti M, Bozhok A, Baronio R, Feyereislova A, Barton C, Valagussa P, Baselga J. Neoadjuvant chemotherapy with trastuzumab followed by adjuvant trastuzumab versus neoadjuvant chemotherapy alone, in patients with HER2-positive locally advanced breast cancer (the NOAH trial): a randomised controlled superiority trial with a parallel HER2-negative cohort. Lancet. 2010;375(9712):377-84. https://doi.org/10.1016/S0140-6736(09)61964-4.

5. von Minckwitz G, Blohmer JU, Costa SD, Denkert C, Eidtmann H, Eiermann W, Gerber B, Hanusch C, Hilfrich J, Huober J, Jackisch C, Kaufmann M, Kümmel S, Paepke S, Schneeweiss A, Untch M, Zahm DM, Mehta K, Loibl S. Responseguided neoadjuvant chemotherapy for breast cancer. J Clin Oncol. 2013;31(29): 3623-30. https://doi.org/10.1200/JCO.2012.45.0940. Epub 2013 Sep 3

6. Wang J, Buchholz TA, Middleton LP, Allred DC, Tucker SL, Kuerer HM, Esteva FJ, Hortobagyi GN, Sahin AA. Assessment of histologic features and expression of biomarkers in predicting pathologic response to anthracycline-based neoadjuvant chemotherapy in patients with breast carcinoma. Cancer. 2002;94(12):3107-14.

7. Lips EH, Mulder L, de Ronde JJ, Mandjes IA, Koolen BB, Wessels LF, Rodenhuis S, Wesseling J. Breast cancer subtyping by immunohistochemistry and histological grade outperforms breast cancer intrinsic subtypes in predicting neoadjuvant chemotherapy response. Breast Cancer Res Treat. 2013;140(1):63-71. https://doi. org/10.1007/s10549-013-2620-0.

8. Liedtke C, Mazouni C, Hess KR, Andre F, Tordai A, Mejia JA, Symmans WF, Gonzalez-Angulo AM, Hennessy B, Green M, Cristofanilli M, Hortobagyi GN, Pusztai L. Response to neoadjuvant therapy and long-term survival in patients with triple-negative breast cancer. J Clin Oncol. 2008;26(8):1275-81. https://doi.org/10.1200/JCO.2007.14.4147.

9. von Minckwitz G, Untch M, Nuesch E, Loibl S, Kaufmann M, Kummel S, Fasching PA, Eiermann W, Blohmer JU, Costa SD, Mehta K, Hilfrich J, Jackisch C, Gerber B, du Bois A, Huober J, Hanusch C, Konecny G, Fett W, Stickeler E, Harbeck N, Muller V, Juni P. Impact of treatment characteristics on response of different breast cancer phenotypes: pooled analysis of the German neo-adjuvant chemotherapy trials. Breast Cancer Res Treat. 2011; 125(1):145-56. https://doi.org/10.1007/s10549-010-1228-x.

10. Zardavas D, Piccart M. Neoadjuvant therapy for breast cancer. Annu Rev Med. 2015;66:31-48. https://doi.org/10.1146/annurev-med-051413-024741.

11. Cortazar P, Zhang L, Untch M, Mehta K, Costantino JP, Wolmark N, Bonnefoi H, Cameron D, Gianni L, Valagussa P, et al. Pathological complete response and long-term clinical benefit in breast cancer: the CTNeoBC pooled analysis. Lancet. 2014;384(9938):164-72. https://doi.org/10.1016/S0140-6736(13)62422-8.

12. Straver ME, Rutgers EJ, Rodenhuis S, Linn SC, Loo CE, Wesseling J, Russell NS, Oldenburg HS, Antonini N, Vrancken Peeters MT. The relevance of breast cancer subtypes in the outcome of neoadjuvant chemotherapy. Ann Surg Oncol. 2010;17(9):2411-8. https://doi.org/10.1245/s10434-010-1008-1.

13. Harry VN, Semple SI, Parkin DE, Gilbert FJ. Use of new imaging techniques to predict tumour response to therapy. Lancet Oncol. 2010;11(1):92-102.

14. Yeh E, Slanetz P, Kopans DB, Rafferty E, Georgian-Smith D, Moy L, Halpern E, Moore R, Kuter I, Taghian A. Prospective comparison of mammography, sonography, and MRI in patients undergoing neoadjuvant chemotherapy for palpable breast cancer. AJR Am J Roentgenol. 2005;184(3):868-77.

15. Humbert O, Riedinger JM, Vrigneaud JM, Kanoun S, Dygai-Cochet I, BerrioloRiedinger A, Toubeau M, Depardon E, Lassere M, Tisserand S, Fumoleau P, Brunotte F, Cochet A. 18F-FDG PET-derived tumor blood flow changes after 1 cycle of neoadjuvant chemotherapy predicts outcome in triple-negative breast cancer. J Nucl Med. 2016;57(11):1707-12.

16. Avril S, Muzic RF Jr, Plecha D, Traughber BJ, Vinayak S, Avril N. ${ }^{18}$ F-FDG PET/ CT for monitoring of treatment response in breast cancer. J Nucl Med. 2016; 57(Suppl 1):34S-9S. https://doi.org/10.2967/jnumed.115.157875. Review

17. Loo CE, Straver ME, Rodenhuis S, Muller SH, Wesseling J, Vrancken Peeters MJ, Gilhuijs KG. Magnetic resonance imaging response monitoring of breast cancer during neoadjuvant chemotherapy: relevance of breast cancer subtype. J Clin Oncol. 2011;29(6):660-6. https://doi.org/10.1200/JCO.2010.31. 1258. Epub 2011 Jan 10
18. Tromberg BJ, Zhang Z, Leproux A, O'Sullivan TD, Cerussi AE, Carpenter PM, Mehta RS, Roblyer D, Yang W, Paulsen KD, Pogue BW, Jiang S, Kaufman PA, Yodh AG, Chung SH, Schnall M, Snyder BS, Hylton N, Boas DA, Carp SA, Isakoff SJ, Mankoff D. ACRIN 6691 investigators. predicting responses to neoadjuvant chemotherapy in breast cancer: ACRIN 6691 trial of diffuse optical spectroscopic imaging. Cancer Res. 2016;76(20):5933-44. Epub 2016 Aug 15.

19. Tran WT, Childs C, Chin L, Slodkowska E, Sannachi L, Tadayyon H, Watkins E, Wong SL, Curpen B, El Kaffas A, Al-Mahrouki A, Sadeghi-Naini A, Czarnota GJ. Multiparametric monitoring of chemotherapy treatment response in locally advanced breast cancer using quantitative ultrasound and diffuse optical spectroscopy. Oncotarget. 2016;7(15):19762-80. https://doi.org/10. 18632/oncotarget.7844.

20. Zhu Q, DeFusco PA, Ricci A Jr, Cronin EB, Hegde PU, Kane M, Tavakoli B, Xu Y, Hart J, Tannenbaum SH. Breast cancer: assessing response to neoadjuvant chemotherapy by using US-guided near-infrared tomography. Radiology. 2013; 266(2):433-42. https://doi.org/10.1148/radiol.12112415. Epub 2012 Dec 21

21. Zhu Q, Wang L, Tannenbaum S, Ricci A Jr, DeFusco P, Hegde P. Pathologic response prediction to neoadjuvant chemotherapy utilizing pretreatment near-infrared imaging parameters and tumor pathologic criteria. Breast Cancer Res. 2014;16(5):456. https://doi.org/10.1186/s13058-014-0456-0.

22. Ueda S, Yoshizawa N, Shigekawa T, Takeuchi H, Ogura H, Osaki A, Saeki T, Ueda Y, Yamane T, Kuji I, Sakahara H. Near-infrared diffuse optical imaging for early prediction of breast cancer response to neoadjuvant chemotherapy: a comparative study using 18F-FDG PET/CT. J Nucl Med. 2016;57(8):1189-95. https://doi.org/10.2967/jnumed.115.167320. Epub 2016 Mar 3

23. Jiang S, Pogue BW, Kaufman PA, Gui J, Jermyn M, Frazee TE, Poplack SP, DiFlorio-Alexander R, Wells WA, Paulsen KD. Predicting breast tumor response to neoadjuvant chemotherapy with diffuse optical spectroscopic tomography prior to treatment. Clin Cancer Res. 2014;20(23):6006-15. https://doi.org/10.1158/1078-0432.CCR-14-1415. Epub 2014 Oct 7

24. Xu C, Vavadi H, Merkulov A, Li H, Erfanzadeh M, Mostafa A, Gong Y, Salehi H, Tannenbaum S, Zhu Q. Ultrasound-guided diffuse optical tomography for predicting and monitoring neoadjuvant chemotherapy of breast cancers: recent progress. Ultrason Imaging. 2016;38(1):5-18. https://doi.org/10.1177/ 0161734615580280. Epub 2015 Apr 16

25. Sajjadi AY, Isakoff SJ, Deng B, Singh B, Wanyo CM, Fang Q, Specht MC, Schapira L, Moy B, Bardia A, Boas DA, Carp SA. Normalization of compression-induced hemodynamics in patients responding to neoadjuvant chemotherapy monitored by dynamic tomographic optical breast imaging (DTOBI). Biomed Opt Express. 2017:8(2):555-69. https://doi. org/10.1364/BOE.8.000555. eCollection 2017 Jan 4.

26. Busch DR, Choe R, Rosen MA, Guo W, Durduran T, Feldman MD, Mies C, Czerniecki BJ, Tchou J, Demichele A, Schnall MD, Yodh AG. Optical malignancy parameters for monitoring progression of breast cancer neoadjuvant chemotherapy. Biomed Opt Express. 2013;4(1):105-21. https:// doi.org/10.1364/BOE.4.000105. Epub 2012 Dec 14

27. Roblyer D, Ueda S, Cerussi A, Tanamai W, Durkin A, Mehta R, Hsiang D, Butler JA, McLaren C, Chen WP, Tromberg B. Optical imaging of breast cancer oxyhemoglobin flare correlates with neoadjuvant chemotherapy response one day after starting treatment. Proc Natl Acad Sci USA. 2011;108:14626-31.

28. Enfield LC, Gibson AP, Hebden JC, Douek M. Optical tomography of breast cancer-monitoring response to primary medical therapy. Target Oncol. 2009;4(3):219-33. https://doi.org/10.1007/s11523-009-0115-z. Epub 2009 Sep.

29. Zhu Q, Tannenbaum S, Hegde P, Kane M, Xu C, Kurtzman SH. Noninvasive monitoring of breast cancer during neoadjuvant chemotherapy using optical tomography with ultrasound localization. Neoplasia. 2008;10(10):1028-40.

30. Cerussi A, Hsiang D, Shah N, Mehta R, Durkin A, Butler J, Tromberg BJ. Predicting response to breast cancer neoadjuvant chemotherapy using diffuse optical spectroscopy. Proc Natl Acad Sci U S A. 2007;104(10):4014-9. Epub 2007 Feb 28

31. Ueda S, Roblyer D, Cerussi A, Durkin A, Leproux A, Santoro Y, Xu S, O'Sullivan TD, Hsiang D, Mehta R, Butler J, Tromberg BJ. Baseline tumor oxygen saturation correlates with a pathologic complete response in breast cancer patients undergoing neoadjuvant chemotherapy. Cancer Res. 2012;72(17): 4318-28. https://doi.org/10.1158/0008-5472.CAN-12-0056. Epub 2012 Jul 9

32. Zhu Q, Ricci A Jr, Hegde P, Kane M, Cronin E, Merkulov A, Xu Y, Tavakoli B, Tannenbaum S. Assessment of functional differences in malignant and benign breast lesions and improvement of diagnostic accuracy by using US-guided diffuse optical tomography in conjunction with conventional US. Radiology. 2016;280(2):387-97. https://doi.org/10.1148/radiol.2016151097. Epub 2016 Mar 2 
33. Zhu Q, Hegde PU, Ricci A Jr, Kane M, Cronin EB, Ardeshirpour Y, Xu C, Aguirre A, Kurtzman SH, Deckers PJ, Tannenbaum SH. Early-stage invasive breast cancers: potential role of optical tomography with US localization in assisting diagnosis. Radiology. 2010;256(2):367-78. https://doi.org/10.1148/ radiol.10091237. Epub 2010 Jun 22

34. Harvey JM, Clark GM, Osborne CK, Allred DC. Estrogen receptor status by immunohistochemistry is superior to the ligand-binding assay for predicting response to adjuvant endocrine therapy in breast cancer. J Clin Oncol. 1999: 17(5):1474-81.

35. Wolff AC, Hammond ME, Hicks DG, Dowsett M, LM MS, Allison KH, Allred DC, Bartlett JM, Bilous M, Fitzgibbons P, Hanna W, Jenkins RB, Mangu PB, Paik S, Perez EA, Press MF, Spears PA, Vance GH, Viale G, Hayes DF. Recommendations for human epidermal growth factor receptor 2 testing in breast cancer: American Society of Clinical Oncology/College of American Pathologists clinical practice guideline update. Am Soc Clin Oncol. 2014; 138(2):241-56. https://doi.org/10.5858/arpa.2013-0953-SA. Epub 2013 Oct 7

36. Ogston KN, Miller ID, Payne S, et al. A new histologic grading system to assess response of breast cancers to primary chemotherapy; prognostic significance and survival. Breast. 2003;12:320-7.

37. Zhu Q, Chen N, Kurtzman SH. Imaging tumor angiogenesis by use of combined near-infrared diffusive light and ultrasound. Opt Lett. 2003;28(5):337-9.

38. Zhu Q, Xu C, Guo P, Aguirre A, Yuan B, Huang F, Castilo D, Gamelin J, Tannenbaum S, Kane M, Hegde P, Kurtzman S. Optimal probing of optical contrast of breast lesions of different size located at different depths by US localization. Technol Cancer Res Treat. 2006;5(4):365-80.

39. Kleinbaum D, Kupper L, Muller K, Nizam A. Applied regression analysis and other multivariable methods. 3rd ed. Belmont: Duxbury Press; 1998.

40. Agresti A. Categorical data analysis. 2nd ed: Wiley; (Section 5.3.2). Somerset, NJ, USA: Wiley; 2002.

41. Pindyck RS, Rubinfeld DL. Econometric models and economic forecasts. 4th ed. (Section 5.2). 2 Pennsylvania Plaza, NY, USA: Irwin/McGraw-Hill; 1998.

42. Zhao Y, Dong X, Li R, Ma X, Song J, Li Y, Zhan D. Evaluation of the pathological response and prognosis following neoadjuvant chemotherapy in molecular subtypes of breast cancer. Onco Targets Ther. 2015;8:1511-21.

43. Symmans WF, Peintinger $F$, Hatzis $C$, et al. Measurement of residual breast cancer burden to predict survival after neoadjuvant chemotherapy. J Clin Oncol. 2007:25:4414-22.

44. Humbert O, Lasserre M, Bertaut A, Fumoleau P, Coutant C, Brunotte F, Cochet A. Pattern of breast cancer blood flow and metabolism assessed using dual-acquisition 18FDGPET: correlation with tumor phenotypic features and pathological response to neoadjuvant chemotherapy. J Nucl Med. 2018; https://doi.org/10.2967/jnumed.117.203075. Epub ahead of print

45. Isumin $Y, X u L$, di Tomaso E, Fukumura D, Jain RK. Tumor biology: herceptin acts as an anti-angiogenic cocktail. Nature. 2002;416:279-80.

46. Tanamai W, Chen C, Siavoshi S, Cerussi A, Hsiang D, Butler J, Tromberg B. Diffuse optical spectroscopy measurements of healing in breast tissue after core biopsy: case study. J Biomed Opt. 2009;14(1):014024.

47. Stein RG, Wollschläger D, Kreienberg R, Janni W, Wischnewsky M, Diessner J, Stüber T, Bartmann C, Krockenberger M, Wischhusen J, Wöckel A, Blettner M, Schwentner L, BRENDA Study Group. The impact of breast cancer biological subtyping on tumor size assessment by ultrasound and mammography - a retrospective multicenter cohort study of 6543 primary breast cancer patients. BMC Cancer. 2016;16:459. https://doi.org/10.1186/ s12885-016-2426-7.

48. Choe R, Konecky SD, Corlu A, Lee K, Durduran T, Busch DR, Pathak S, Czerniecki BJ, Tchou J, Fraker DL, Demichele A, Chance B, Arridge SR, Schweiger M, Culver JP, Schnall MD, Putt ME, Rosen MA, Yodh AG. Differentiation of benign and malignant breast tumors by in-vivo threedimensional parallel-plate diffuse optical tomography. J Biomed Opt. 2009; 14(2):024020.

Ready to submit your research? Choose BMC and benefit from:

- fast, convenient online submission

- thorough peer review by experienced researchers in your field

- rapid publication on acceptance

- support for research data, including large and complex data types

- gold Open Access which fosters wider collaboration and increased citations

- maximum visibility for your research: over $100 \mathrm{M}$ website views per year

At BMC, research is always in progress.

Learn more biomedcentral.com/submissions 